\title{
Laboratory experiments and models of diffusive emplacement of ground ice on Mars
}

\author{
Troy L. Hudson, ${ }^{1}$ Oded Aharonson, ${ }^{1}$ and Norbert Schorghofer ${ }^{2}$ \\ Received 20 March 2008; revised 24 July 2008; accepted 4 November 2008; published 23 January 2009.
}

[1] Experiments demonstrate for the first time the deposition of subsurface ice directly from atmospheric water vapor under Mars surface conditions. Deposition occurs at pressures below the triple point of water and therefore in the absence of a bulk liquid phase. Significant quantities of ice are observed to deposit in porous medium interstices; the maximum filling fraction observed in our experiments is $\sim 90 \%$, but the evidence is consistent with ice density in pore spaces asymptotically approaching $100 \%$ filling. The micromorphology of the deposited ice reveals several noteworthy characteristics including preferential early deposition at grain contact points, complete pore filling between some grains, and captured atmospheric gas bubbles. The boundary between icebearing and ice-free soil, the "ice table," is a sharp interface, consistent with theoretical investigations of subsurface ice dynamics. Changes of surface radiative properties are shown to affect ice table morphology through their modulation of the local temperature profile. Accumulation of ice is shown to reduce the diffusive flux and thus reduce the rate of further ice deposition. Numerical models of the experiments based on diffusion physics are able to reproduce experimental ice contents if the parameterization of growth rate reduction has expected contributions in addition to reduced porosity. Several phenomena related to the evolution of subsurface ice are discussed in light of these results, and interpretations are given for a range of potential observations being made by the Phoenix Scout Lander.

Citation: Hudson, T. L., O. Aharonson, and N. Schorghofer (2009), Laboratory experiments and models of diffusive emplacement of ground ice on Mars, J. Geophys. Res., 114, E01002, doi:10.1029/2008JE003149.

\section{Introduction}

[2] Communication between subsurface ice and the atmosphere is an important component of Mars's water cycle history [Mellon and Jakosky, 1995; Mellon et al., 2004]. This buried ice comprises a vast reservoir which may hold a significant fraction of the total water inventory that is able to exchange with the atmosphere and polar caps [Carr, 1996]. Mellon and Jakosky [1993] have predicted that ice can accumulate in soil pores from atmospherically derived water vapor. We reproduce this phenomenon at Mars-like conditions of low temperature and low pressure for the first time in laboratory experiments.

[3] In the present climate, ice is stable in the shallow subsurface in latitudes poleward of about 60 degrees as predicted by models [e.g., Leighton and Murray, 1966; Mellon and Jakosky, 1993; Schorghofer and Aharonson, 2005], and confirmed by observations [e.g., Boynton et al., 2002]. It is hypothesized that the stability regions occupied higher latitudes when Mars's obliquity was low, and were

\footnotetext{
${ }^{1}$ Division of Geological and Planetary Sciences, California Institute of Technology, Pasadena, California, USA.

${ }^{2}$ Institute for Astronomy, University of Hawai' $i$ at Mānoa, Honolulu, Hawaii, USA.
}

Copyright 2009 by the American Geophysical Union. 0148-0227/09/2008JE003149\$09.00 global when the obliquity was high [Mellon and Jakosky, 1995; Head et al., 2003]. The close agreement between present-day climate and model predictions is taken as evidence that the majority of the current subsurface ice is at or near its diffusive equilibrium depth with respect to the mean annual atmospheric vapor content. The observed hydrogen abundances in the middle to high latitudes, converted to water equivalent hydrogen, indicate that a significant percentage (at least 60\%) of the mass of the upper meter of the regolith is ice [Prettyman et al., 2004]. Litvak et al. [2006] and Feldman et al. [2007], however, suggest that the ice content of the ice-rich layer depends on latitude, and the ice content is consistent with the existence of interstitial ice in some latitude range. While some of this hydrogen may indicate pure ice layers deposited as precipitation and subsequently buried, much of the buried ice may have been derived directly from atmospheric water vapor [Schorghofer, 2007].

[4] As the orbital and axial parameters of the planet change, the ice responds to changing temperatures and humidities, and the time scale of this response is modulated by the diffusivity of the regolith [Mellon and Jakosky, 1995; Hudson et al., 2007]. Diffusion coefficients for a variety of materials, including salt crusts, sand, dust, and mixtures with a wide particle size range, have been measured under simulated Mars surface conditions and have been found to lie within a range of $\sim 0.2-5 \mathrm{~cm}^{2} \mathrm{~s}^{-1}$ [Hudson et al., 2007; 
Hudson and Aharonson, 2008]. As discussed by Hudson et al. [2007], this range would permit an ice table adjustment on the order of meters within one $120 \mathrm{ka}$ obliquity cycle. The minor variations in obliquity over the past few hundred thousand years would have permitted even regions with relatively low diffusivities to adjust.

[5] The rate at which ice accumulates in pore spaces as a function of time is obtained through conservation of mass and an expression for the vapor flux. The vapor flux is taken to be proportional to the saturation vapor density gradient,

$$
J_{1} \propto \frac{\partial \rho_{1}}{\partial z},
$$

where $J_{1}$ is the diffusive mass flux of $\mathrm{H}_{2} \mathrm{O}$ and $\rho_{1}$ is the mass density of water vapor. In a dry porous medium these quantities define a proportionality factor $D$, the diffusion coefficient, which thus gives Fick's First Law. Since the investigation here focuses on media with active sublimation and evaporation from pore walls, the relationship between vapor flux and the vapor density gradient may be different from that experienced in an inert medium. This fact, and a pore geometry which changes with time, prompts an definition for the proportionality factor which uses the free gas diffusion coefficient, $\mathcal{D}_{12}$, times an obstruction factor related to the porosity $\phi$ and tortuosity $\tau$ of the medium: $D=$ $f(\phi, \tau) \mathcal{D}_{12}$. This is analogous to the definition of $D$ in Fick's First Law, but may be different in its specific behavior and the relationship between $D$ and the pore space geometry.

[6] Conservation of mass, $\partial \sigma / \partial t+\partial J / \partial z=0$, and the definition of $D$ given above lead to an expression for the accumulation of ice in pore spaces as a function of time:

$$
\frac{\partial \sigma}{\partial t}=-\frac{\partial J_{1}}{\partial z}=\frac{\partial}{\partial z}\left(D \frac{\partial \rho_{1}}{\partial z}\right)
$$

The quantity $\sigma$ is the density of ice relative to total volume (i.e., the total volume occupied both by voids and by regolith particles, as opposed to $\rho_{1}$, which is a density relative to the gas-filled pore volume). The quantities in equation (2) known or directly measurable in the experiments described below are the ice accumulation rate, $\partial \sigma / \partial t$, and the vapor density gradient. These can be used to compute values of the quantity $D$. It is important to stress again that $D$ in a medium with walls coated in ice, acting as both source and sink to maintain saturated pore gas, may behave differently than the diffusion coefficient computed for an ice-free medium with the same geometry.

[7] As per equation (2), ice accumulates in a permeable medium as a humidity gradient supplies water molecules. On Mars, such a gradient can exist between a warm and humid but unsaturated atmosphere and a cold and therefore saturated location in the subsurface, the flux directed toward the already saturated cold spot. This infilling phenomenon has first been modeled numerically by Mellon and Jakosky [1993], and for Mars environments typically implies the accumulation of ice in pore spaces beneath a dry layer.

[8] The presence of ice changes the thermal properties of the regolith by increasing the thermal conductivity of an unconsolidated porous medium [Paige, 1992]. But the formation of subsurface ice may also reduce the diffusivity as a consequence of pore space reduction. As the pore space is reduced, a regolith with an initially large pore diameter will transition from Fickian diffusion, where moleculemolecule collisions dominate, to Knudsen diffusion wherein most collisions are between molecules and the pore walls. It has been suggested [Mellon and Jakosky, 1995] that ice deposited from the vapor phase may completely choke off vapor transport paths. Since subsurface ice has been shown to grow most rapidly near its equilibrium depth [Mellon and Jakosky, 1995; Schorghofer and Aharonson, 2005], choking would occur first at this level and inhibit diffusion to greater depths.

[9] Subfreezing (i.e., bulk liquid-free) vapor diffusion in soils finds further application in the evolution of subsurface ice on Earth. The longevity of ice in cold, arid locations such as the Dry Valleys of Antarctica constrains time scales of terrestrial climate change [Sugden et al., 1995; Hindmarsh et al., 1998]. Many sites in the McMurdo Dry Valleys have dry permafrost within the upper meter of the glacial till comprising the valley floor [Bockheim, 2002]. The depth and extent of this ice may be controlled in part by vapor diffusion processes, particularly where precipitation and mean annual temperatures are low and liquid water is largely absent [Hindmarsh et al., 1998; McKay et al., 1998; Schorghofer, 2005]. While the studies in this work are motivated by Mars, they may also be relevant for such localities on Earth. However, situations on Earth where no melting is involved in ice evolution are rare and localized, leading to a dearth of theoretical, experimental, and observational permafrost research focused on ice deposition via gaseous diffusion.

[10] We perform experiments to examine the process of ice deposition in porous media under Mars-like surface conditions. The resulting ice content profiles reveal the presence, depth, and sharpness of the developed ice table. A variety of atmospheric humidity regimes and experiment durations are employed to provide information about the rate at which the pores fill. Long-duration experiments allow us to approach the ice-filled state. A numerical model of ice deposition is run with experimental temperatures, humidities, and ice-free diffusivity as inputs. This model incorporates parameterizations of pore space reduction and comparisons between the model and experimental data allow these schema to be evaluated.

\section{Experiments}

\subsection{Parameter Choices}

[11] The atmosphere of present-day Mars has low absolute humidity, and the upper levels of the regolith are subject to ice-filling vapor density gradients on the order of $10^{-6} \mathrm{~kg} \mathrm{~m}^{-4}$. Under these conditions, the maximum rate of ice deposition would fill a regolith with $\sim 40 \%$ porosity in approximately $10^{5}$ years. Obviously, such time scales are impractical for measurable ice contents to be developed in the laboratory. To enhance the deposition rate, we employ both a more humid atmosphere and a stronger vapor density gradient than found on Mars today, which allow useful experiments to be run in hours or days rather than years. We discuss how these choices affect the physics of diffusion (including an increased advection and thermodiffusion contribution) in section 5.1. 


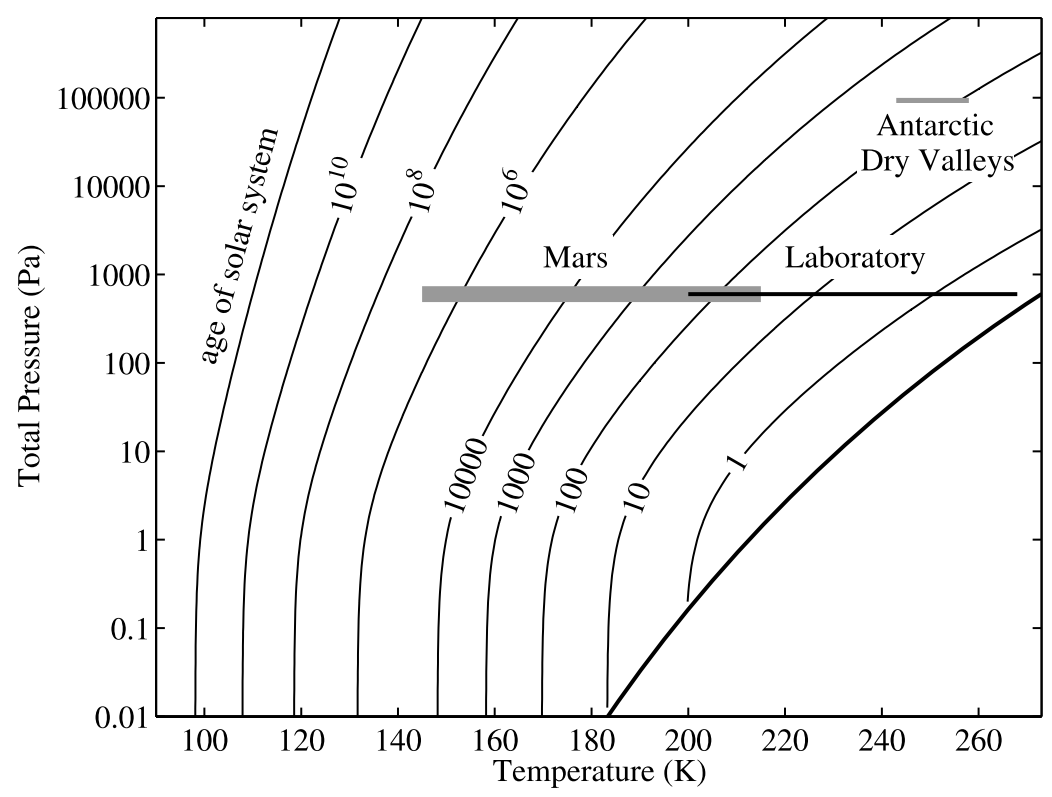

Figure 1. Contours of time in days to deposit a quantity of ice equivalent to $42 \%$ porosity for a temperature gradient of $13 \mathrm{~K} \mathrm{~cm}^{-1}$ using the simple model developed in section 2.1 . This model assumes a time-invariant $D$ and extrapolates initial growth rates and therefore obtains a lower limit on filling times. The diffusivity of the simulant (500 $\mu \mathrm{m}$ glass beads) is extrapolated to appropriate temperatures and pressures using the Fickian formalism, which gives $D \propto p_{0}{ }^{-1} T^{3 / 2}$, where $p_{0}$ is the total pressure. The heavy black curve is the saturation vapor line. The range of conditions on the surface of Mars, in the Dry Valleys of Antarctica [Doran et al., 2002], and our experimental setup are indicated by horizontal bars. Note that the assumed temperature gradient for the laboratory experiments does not apply in these environments and that the growth rates would be slower.

[12] The surface of Mars experiences substantial diurnal and annual variations in surface temperature. The mean annual vapor pressure experienced by ice within the top few thermal skin depths will be biased toward higher pressures at shallower depths because of the nonlinear dependence of vapor pressure on temperature. This gives rise to a gradient which will pump water vapor into the subsurface if the atmospheric vapor density is above that at equilibrium with ice at depth. Rather than employing a varying surface temperature to create a gradient, the laboratory setup instead uses a constant thermal gradient across the sample. The resulting large gradient in vapor density permits the rapid growth of ice.

[13] An estimate of the rate of ice growth may be obtained by assuming $D$ is independent of depth, and thus $\partial \sigma / \partial t$ is proportional to $\partial^{2} \rho_{1} / \partial z^{2}$, according to equation (2). Using the Clausius-Clapeyron expression for vapor pressure, the second derivative of the vapor density gradient may be approximated by

$$
\frac{\partial^{2}}{\partial z^{2}}\left(\frac{p_{s v}}{T}\right) \approx \frac{p_{s v}}{T}\left[\left(\frac{H}{R T}\right)^{2}-4 \frac{H}{R T}+2\right]\left(\frac{1}{T} \frac{\Delta T}{\Delta z}\right)^{2}
$$

for a linear temperature gradient. Here, $H$ is the enthalpy of sublimation, $R$ the universal gas constant, and $p_{s v}$ is saturation vapor pressure in equilibrium with ice. This simple scheme assumes that $D$ is also independent of time.

[14] Converting equation (3) to density using the ideal gas law, the initial growth rate may be determined. This is then extrapolated to obtain a minimum time to complete pore filling. Since the temperature gradient enters quadratically in equation (3), a strong temperature gradient is clearly desirable for experiments.

[15] Figure 1 plots contours of the number of days required to achieve complete pore filling using $\Delta T / \Delta z=$ $13 \mathrm{~K} \mathrm{~cm}^{-1}$ and a diffusivity of $4.7 \mathrm{~cm}^{2} \mathrm{~s}^{-1}$. Figure 1 reveals a parameter window where significant ice volume accumulates within days. The pore filling times are underestimates for any given set of conditions since $D$ is assumed to be constant in time, but it clearly shows the time advantage gained by operating at higher temperatures. Lower pressure accelerates the growth because of higher diffusion coefficients, but once the Knudsen regime is reached, the mean free path of molecules no longer increases with decreasing pressure. Overlying the contours are the conditions of mean annual temperature and pressure on Mars's surface today, the conditions in our experimental setup, and the conditions in the Dry Valleys of Antarctica.

\subsection{Environmental Control}

[16] Experiments were conducted at the Mars Simulation and Ice Laboratory at Caltech. A custom built stainless steel vacuum chamber from LACO Technologies was used to achieve Mars-like pressures and a controlled humidity environment. The environmental chamber is a vertical cylinder with a removable lid, viton O-ring seals, and multiple electrical and fluid feed throughs. The volume of the chamber is $2.3 \times 10^{-2} \mathrm{~m}^{3}$. Important environmental parameters which are controlled through the course of an 


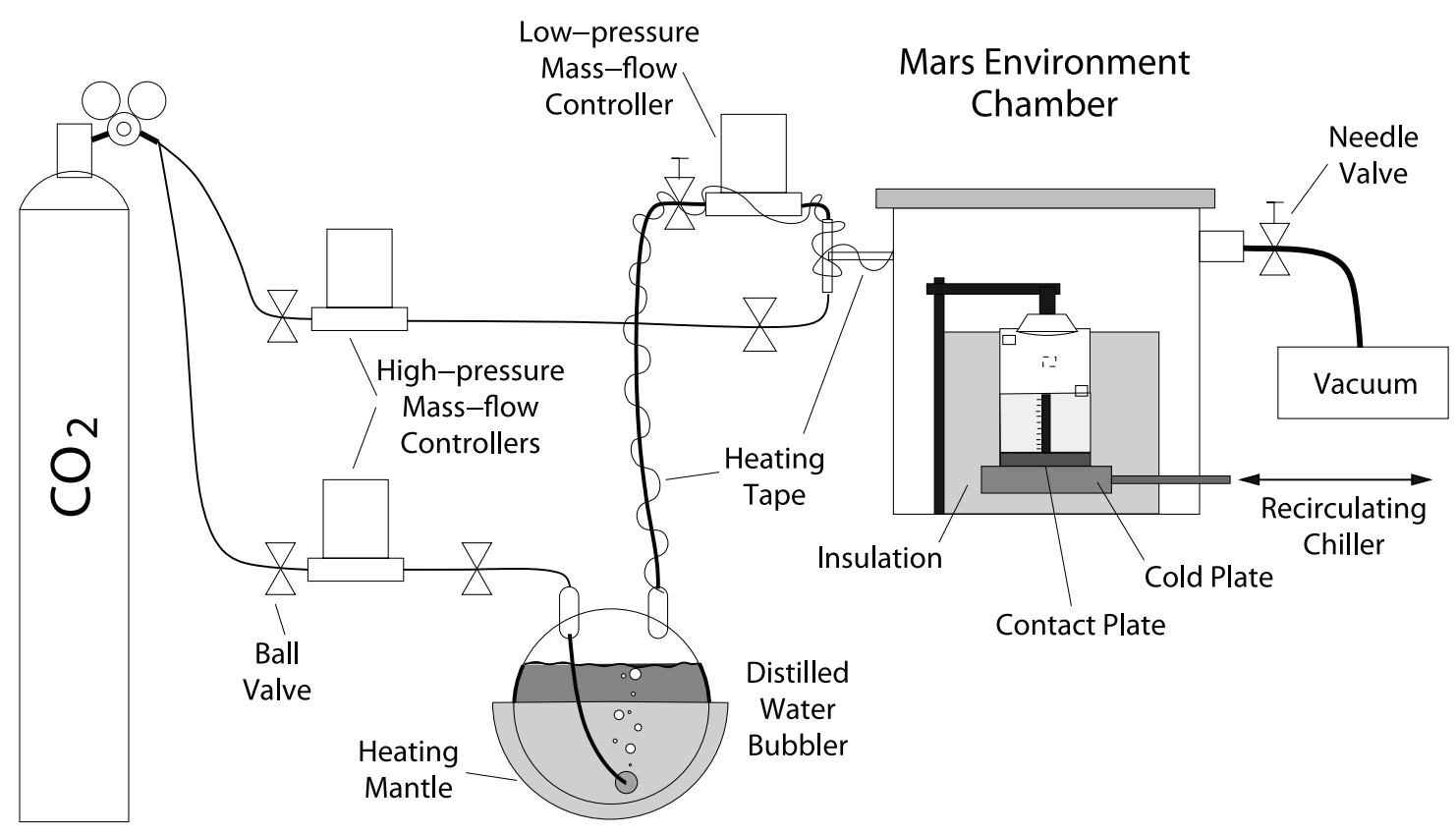

Figure 2. Schematic of experimental setup highlighting gas humidification and delivery and pressure control. The sample container within the environment chamber at right is expanded and annotated in Figure 3. The data logger and electrical interface connections are not shown.

experiment are the chamber pressure, atmospheric vapor density, and the top and bottom boundary temperatures of the sample column. These are maintained through 5 simultaneously operating Proportional-Integral-Derivative (PID) control loops.

[17] A schematic of the complete experimental setup is presented in Figure 2. Several interacting systems work to control the atmospheric vapor density and total pressure in the chamber. A vacuum pump continuously evacuates the chamber during an experiment and the pumping speed can be manually adjusted by a needle valve in the vacuum line. Evacuation is compensated by input of a controlled mixture of dry and humid $\mathrm{CO}_{2}$. To create humid $\mathrm{CO}_{2}$, dry $\mathrm{CO}_{2}$ is passed through a mass flow controller (PID loop 1) and a sparger into a $5 \mathrm{~L}$ vessel of distilled, deionized water. The temperature of the water in this bubbler is maintained at $38^{\circ}$ via PID control (loop 2) of a hemispherical heating mantle. The gas output of the bubbler leads to the main chamber. Pressure in the bubbler is forced down by the evacuation of the main chamber but is maintained at approximately 60 mbar by PID loop (3) actuation of the dry gas input which feeds the bubbler. By maintaining a constant bubbler temperature and pressure, the vapor content of the head space gas in the bubbler is maintained to within about $10 \%$. The bubbler pressure is kept slightly bellow the boiling point of water so that the head space gas has a high water content (typically $\sim 85-90 \%$ relative humidity at $30^{\circ} \mathrm{C}$ ). This humid gas is fed into the main chamber through a mass flow controller optimized for regulating low-pressure flows. This MFC is also under PID control (loop 4) where a humidity sensor (described below) provides input to the loop by measuring the vapor density of water at the sample surface. A heating tape around the gas line from the bubbler prevents water from condensing before it reaches the chamber. A third mass flow controller regulates the input of dry gas into the main chamber (loop 5). The low-pressure MFC control loop (4) first adjusts the input of humid gas to achieve the desired chamber vapor density. The dry gas loop (5) then brings the total chamber pressure to 6 mbar. Pressure feedback between these two loops is rapid and settles within $\sim 5$ min to a steady state which can be tuned so the controllers are far from saturation.

[18] In our experiment, we attempt to remove as much water from the sample as possible before introducing subzero temperatures. Porous media simulant which has been kept dry in an oven at $130^{\circ} \mathrm{C}$ is cooled to approximately $50^{\circ} \mathrm{C}$ in a desiccator and then poured into place in the sample chamber. The chamber is then sealed and pumped down to $600 \mathrm{~Pa}$ and maintained with dry $\mathrm{CO}_{2}$. The sample surface is maintained at $50^{\circ} \mathrm{C}$ by a halogen lamp until the measured vapor density drops below $0.1 \mathrm{~g}$ $\mathrm{m}^{-3}$, usually several hours. Any water remaining in the chamber when the cold plate is activated is quickly gettered onto the lowest level of the soil. The low vapor densities measured in the cold, dry chamber suggest that any ice present when the chamber is opened to humid gas should only persist in the bottom $1-2 \mathrm{~cm}$ of the sample where the soil is $\$ 220 \mathrm{~K}$, and we later show that this amount of ice is negligible.

[19] The sample column is chilled from its base via a copper cold plate. A methanol recirculating chiller acts to keep the cold plate at $183 \pm 1 \mathrm{~K}$. Thermal losses through silicone grease connecting the cold plate and the contact plate attached to the sample caddy results in a sample base temperature of approximately $193 \pm 2 \mathrm{~K}$. The cold plate, cooling lines, and all other cold surfaces in the chamber are coated with polyurethane foam to slow heat conduction and prevent gettering of atmospheric water in locations other than the soil column. The surface of the sample is maintained at $268 \mathrm{~K}$ via another independent PID loop 


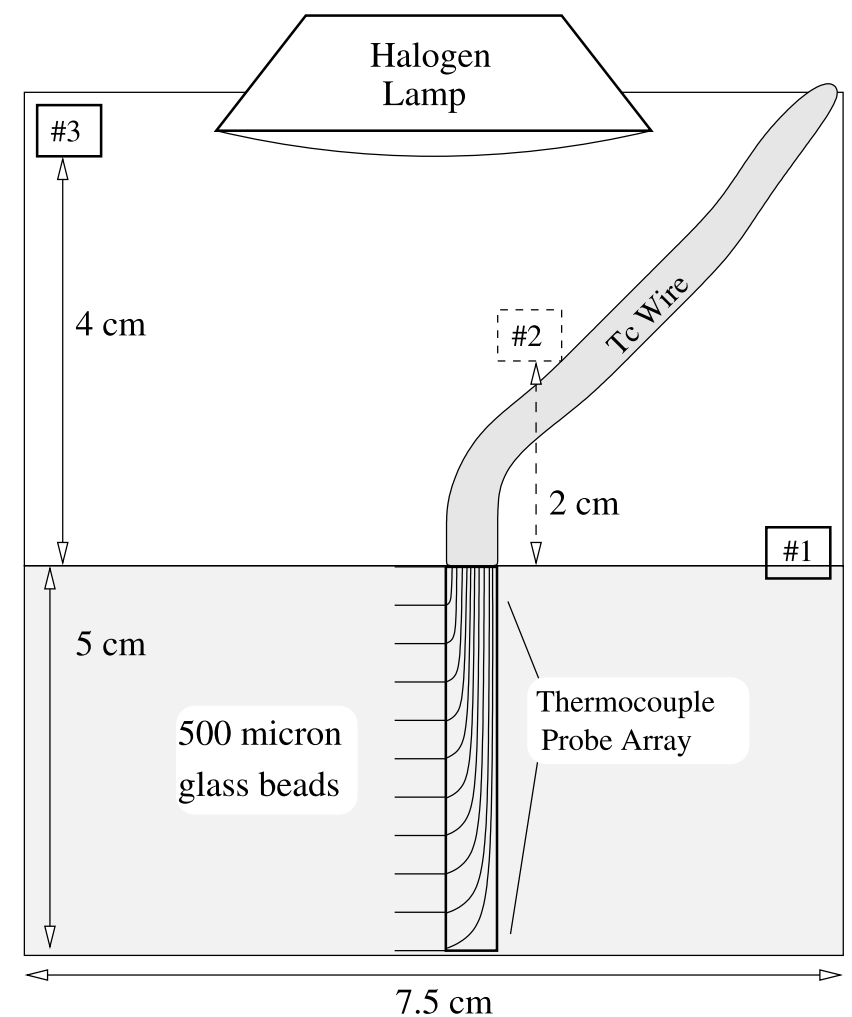

Figure 3. Schematic of sample container. Numbered boxes are positions of RH/RTD sensors. Sensor 1 has its sensing face buried $\sim 2 \mathrm{~mm}$ beneath the sample surface. Sensor 1 and sensor 2 (against the back wall of the cylindrical caddy) are directly illuminated by the lamp. Sensor 3 is not directly illuminated but is in close proximity to the lamp reflector. Thermocouple leads are wrapped in aluminum foil and cast a shadow over part of the sample surface.

controlling a halogen lamp and reflector placed approximately $6 \mathrm{~cm}$ above the sample surface. The input for the lamp controller is the surface temperature as measured by the uppermost thermocouple of the sample thermal probe described in the next section.

\subsection{Sensors}

[20] The pressure in the main chamber is monitored using an MKS Baratron 626 capacitance manometer with a fullscale range of 10 torr. The pressure in the bubbler is monitored with an MKS 902 series piezo-transducer, with a full range of 1000 torr.

[21] Water content of the chamber atmosphere is monitored using RH/RTD sensors (Honeywell model HIH-4602-C) which simultaneously report relative humidity $(\mathrm{RH})$ and ambient temperature at the sensor via a platinum resistance temperature device (RTD). The ITS-90 formulation [Hardy, 1998] is used to convert the relative humidity into a partial pressure of water. Vapor density is calculated via the ideal gas law using the measured RTD temperature.

[22] Temperature as a function of depth is monitored with a custom temperature probe. The probe consists of $11 \mathrm{~T}$ type thermocouples which protrude at half-centimeter intervals from a perforated plastic tube. The sensing ends of the thermocouples are approximately one half centimeter from the plastic body of the sensor assembly and are held in place with a fin of kapton tape which wraps around the sensor body. This arrangement minimizes the effects of vertical heat transport through the sensor body to the thermocouple heads and allows each thermocouple to be in close equilibrium with the local thermal environment.

[23] The data from an experiment are recorded with a National Instruments Compact DAQ-9172 data logger. All process variables are monitored throughout an experimental run at approximately 3 -s intervals. Averaging of these data is performed every minute and the averages are logged. Since all process variables change slowly, this does not compromise the integrity of the data set.

\subsection{Sample Preparation and Method}

[24] The porous medium used in all experiments is composed of soda lime glass beads obtained from AGSCO Corporation (mesh size $30-40$ ). The mean particle size is $500 \mu \mathrm{m}$, no more than $10 \%$ larger than $700 \mu \mathrm{m}$ and no more than $20 \%$ smaller than $400 \mu \mathrm{m}$ (AGSCO technical data sheet). The density of the glass is $2.5 \pm 0.1 \mathrm{~g} \mathrm{~cm}^{-3}$. The diffusivity for gas moving through a bed of these beads were measured using the techniques of Hudson et al. [2007] under $586 \mathrm{~Pa} \mathrm{CO}_{2}$ at $250 \mathrm{~K}$, and was found to be $4.7 \pm$ $0.5 \mathrm{~cm}^{2} \mathrm{~s}^{-1}$. The glass beads' size place them in the sedimentological category of "coarse sand."

[25] The sample caddy is a thin-walled squat plastic cylinder $\sim 7.5 \mathrm{~cm}$ in diameter, the base of which is a snugly fit brass contact plate (see Figure 3). Oven-dried simulant is poured into the caddy to a depth of $5 \mathrm{~cm}$. The temperature probe is then inserted through the sample and the relative humidity sensor is positioned such that it penetrates the surface of the soil by a few millimeters. The tops and exposed wires of the sensors are wrapped in aluminum foil to reduce direct heating by the halogen lamp and to make any heating effects more uniform among the thermocouple wires.

[26] The chamber is sealed and evacuated to $600 \mathrm{~Pa}$ and maintained at this pressure with input of dry $\mathrm{CO}_{2}$ for several hours to remove residual water. The recirculating chiller is then activated and the sample is cooled for at least $6 \mathrm{~h}$ until the sample temperature profile reaches steady state. At all subsequent times the temperature profile is approximately linear, but changes in sample thermal conductivity due to ice deposition and heat losses through the caddy walls result in minor deviations following exposure to humid gas. Once thermally equilibrated, the humid $\mathrm{CO}_{2}$ line is opened and the humidity stabilizes at the desired value.

[27] Figure 4 shows the temperature as a function of depth within a representative simulant during the course of an experiment. Within $5 \mathrm{~min}$ of exposure, the temperature profile changes rapidly between its preexposure (dry) state and a profile which is on average $\sim 10 \mathrm{~K}$ warmer at depths greater than $1 \mathrm{~cm}$ from the temperature-controlled top surface. Following this rapid initial shift to a warmer profile, the subsequent evolution as the ice content of the sample increases is moderate, reaching at most $\sim 5 \mathrm{~K}$ at any depth in the sample. Depths between 0.5 and $3 \mathrm{~cm}$ from the surface exhibit a steady decrease in temperature following the temperature spike during exposure, but deeper regions appear to take longer to reach their maximum temperatures.

[28] The latent heat of condensation can explain the rapid temperature increase at the beginning of exposure and the 


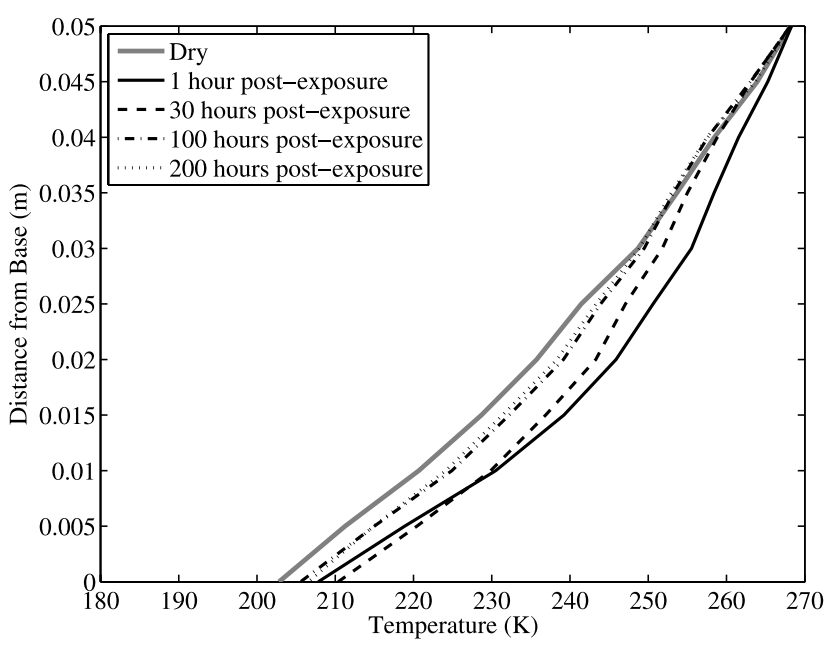

Figure 4. A time series of temperature profiles through the sample of 25 September 2007, exposed to humid gas for $330 \mathrm{~h}$. The heavy gray line is the temperature profile prior to exposure while the sample was dry. Subsequent temperature profiles are given for $1,30,100$, and $200 \mathrm{~h}$ after exposure began.

continued elevated temperatures above the dry-sample baseline. The maximum ice deposition rate exhibited by these experiments is at most $3 \times 10^{-3} \mathrm{~kg} \mathrm{~m}^{-3} \mathrm{~s}^{-1}$ (see section 3.5). Given a heat capacity of ice of $\sim 1960 \mathrm{~J} \mathrm{~K}^{-1}$ $\mathrm{kg}^{-1}$, and a latent heat of condensation from vapor of $2.833 \times 10^{6} \mathrm{~J} \mathrm{~kg}^{-1}$, this rate could produce up to $17 \mathrm{~K}$ of heating in $1 \mathrm{~h}$. This high rate of deposition is short lived, and the heat is rapidly conducted away by the cold sample base, but may account for the $10 \mathrm{~K}$ rise seen throughout most of the sample upon exposure. Reduced deposition rates at subsequent times reduce the difference between the ice-bearing (also ice-depositing) temperature profile and the profile of the ice-free simulant. The effect on the thermal profile of increased thermal conductivity in an ice-bearing sample is offset by the increasing lamp illumination required to maintain the surface temperature at $268 \mathrm{~K}$.

[29] At the conclusion of the experiment, the chamber is purged and the ice-bearing sample and caddy are extracted to a $260 \mathrm{~K}$ freezer. The sample caddy is affixed to a custom extrusion device and the sample is sliced horizontally at half-centimeter intervals. Each layer is removed by scraping and brushing (in the case of poorly consolidated soils) or sawing (in the case of ice-rich soils), and collected in a glass beaker. A funnel is used to capture both bulk material and kerf from the sawing process, minimizing sample loss during analysis. Samples from each layer are immediately weighed, giving a total mass (ice + simulant), $m_{t}$. Beakers of ice-bearing sample are then placed into an oven at $390 \mathrm{~K}$ for a minimum of $24 \mathrm{~h}$. The dried samples are cooled to freezer temperatures in a desiccator. They are weighed again at the same temperature as the ice-bearing samples to obtain the dry simulant mass per layer, $m_{s}$.

\subsection{Data Analysis}

[30] Difference in mass before and after baking is the gravimetric water content. The combined height of all samples measured at the conclusion of the experiments is within $\sim 1 \mathrm{~mm}$ of the preexperiment values, indicating negligible bulk expansion. For $500 \mu \mathrm{m}$ glass beads, the bulk density is determined to be $\rho_{\text {bulk }}=1.46 \pm 0.01 \mathrm{~g} \mathrm{~cm}^{-3}$, and the ice-free porosity is $42 \%$. Layer thicknesses are calculated from the measured mass of dry soil as $\delta z=m_{s} /$ $\left(\rho_{\text {bulk }} A\right)$, where $\delta z$ is the thickness of layer $z$, and $A$ is the area of the sample caddy.

[31] Over the range of temperature encountered in this experiment, the density of bulk ice varies by $\sim 1 \%$, and we assume that all deposited ice has a density of $\rho_{\text {ice }}=918 \mathrm{~kg}$ $\mathrm{m}^{-3}$. The mass of water per layer allows the calculation of ice density relative to total volume, $\sigma=m_{w} / v=\left(m_{t}-m_{s}\right) /$ $(\delta z A)=\rho_{\text {bulk }}\left(m_{t} / m_{s}-1\right)$, where $m_{w}$ and $v$ are the mass of ice and the total volume in a given layer. The maximum possible ice density relative to total sample volume is $\sigma_{0}$ $=\phi_{0} \rho_{\text {ice}}$, where $\phi_{0}$ is the ice-free porosity and $\rho_{\text {ice }}$ is the density of pure ice, and thus the filling fraction is $\sigma / \sigma_{0}$.

[32] A control experiment was run to test the degree of ice accumulation during sample analysis. A sample was prepared in the same manner as other samples, but was never exposed to a humid chamber atmosphere. The amount of water collected by the sample during analysis is less than $0.4 \%$ filling fraction, negligible compared to all nonzero experiment times.

\section{Results}

[33] Experiment durations, target surface vapor densities and observed ice table depths are given in Table 1.

\subsection{Diffusive Ice Morphology}

[34] The deposition of ice in porous media at conditions below the triple point of water experimentally demonstrates ice vapor diffusion and deposition in the absence of a liquid phase. Significant deposition occurs at all sample depths, indicating vapor diffusion as the primary mechanism for $\mathrm{H}_{2} \mathrm{O}$ transport. Though quasi-liquid films may be present under these conditions in the shallow $(\mathrm{T}>250 \mathrm{~K})$

Table 1. List of Experiments ${ }^{\mathrm{a}}$

\begin{tabular}{cccc}
\hline Duration (hours) & $\rho_{1}\left(\mathrm{~g} \mathrm{~m}^{-3}\right)$ & Max Filling $(\%)$ & $z_{\text {ice }}(\mathrm{cm})$ \\
\hline 0 & 1.60 & $0 \pm 1$ & 0 \\
6 & 1.60 & $11 \pm 2$ & 1.1 \\
12 & 1.60 & $15 \pm 3$ & 0.6 \\
18 & 1.60 & $21 \pm 2$ & 0.2 \\
24 & 1.60 & $29 \pm 3$ & 0.1 \\
24 & 1.60 & $29 \pm 3$ & 0.1 \\
24 & 1.60 & $26 \pm 3$ & 0 \\
24 & 1.60 & $29 \pm 3$ & 0 \\
48 & 1.60 & $42 \pm 3$ & 0 \\
48 & 1.60 & $43 \pm 3$ & 0 \\
72 & 1.60 & $54 \pm 4$ & 0 \\
72 & 1.60 & $55 \pm 4$ & 0 \\
96 & 1.60 & $64 \pm 5$ & 0 \\
96 & 1.60 & $76 \pm 4$ & 0 \\
98 & 1.60 & $71 \pm 9$ & 0 \\
120 & 1.60 & $65 \pm 4$ & 0 \\
122 & 1.60 & $71 \pm 5$ & 0 \\
331 & 1.60 & $75 \pm 5$ & 0 \\
530 & 1.60 & $90 \pm 4$ & 0 \\
24 & 0.60 & $10 \pm 2$ & 2.2 \\
24 & 0.85 & $13 \pm 2$ & 1.8 \\
24 & 1.10 & $21 \pm 2$ & 0.5 \\
24 & 1.35 & $26 \pm 3$ & 0.1 \\
\hline
\end{tabular}

\footnotetext{
${ }^{\mathrm{a}} z_{\text {ice }}$ is ice table depth; a value of 0 denotes surface ice.
} 

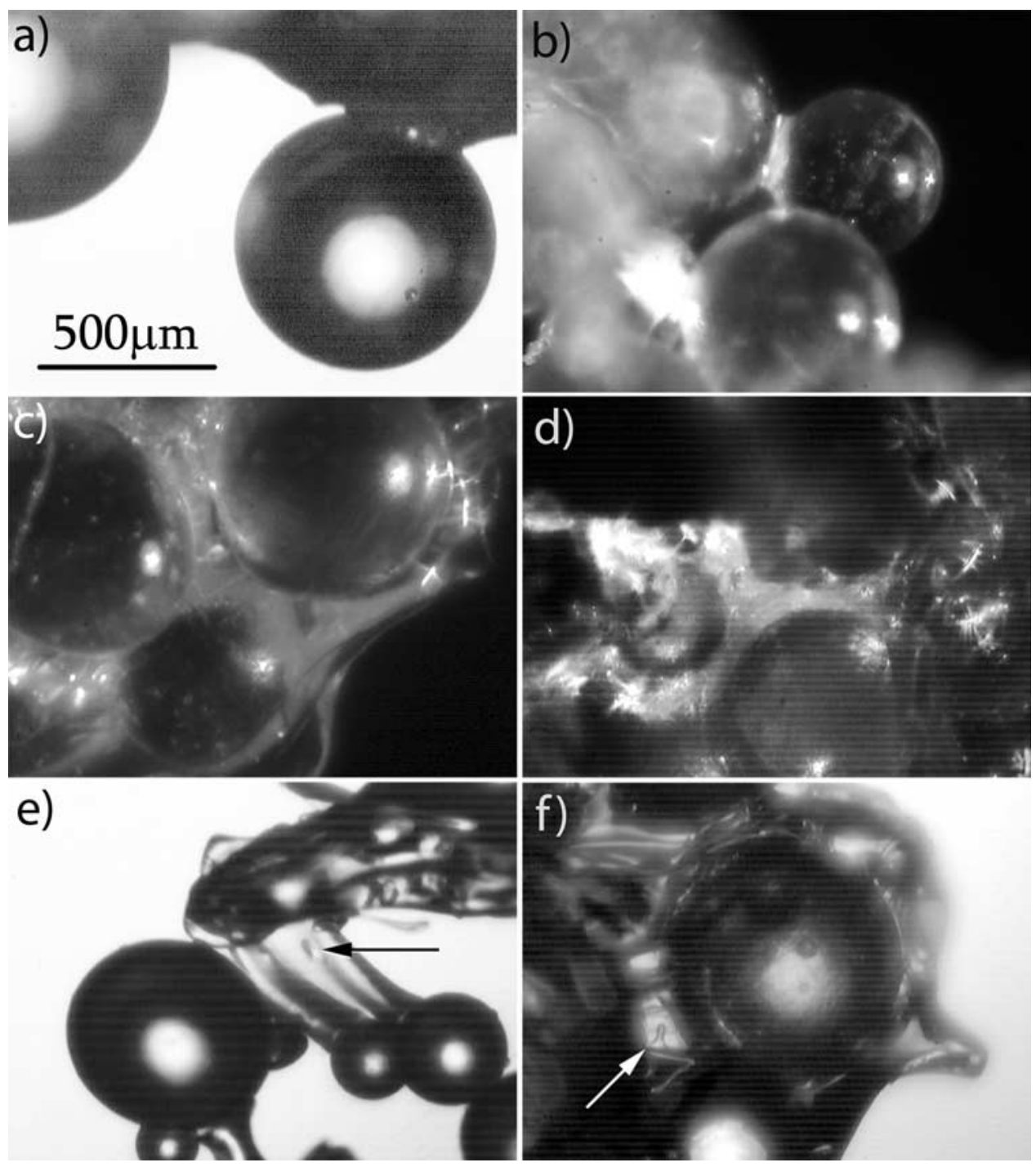

Figure 5. Images of the porous medium (500 $\mu \mathrm{m}$ glass beads) after exposure to experimental conditions. Figures 5a, 5e, and $5 \mathrm{f}$ are back lit; the scale bar applies to all images. (a-c) From the $331 \mathrm{~h}$ experiment and $(\mathrm{d}-\mathrm{f})$ from a $72 \mathrm{~h}$ experiment. All were performed at a nominal vapor density, monitored by sensor 1 , of $1.6 \mathrm{~g} \mathrm{~m}^{-3}$. Figures $5 \mathrm{a}$ and $5 \mathrm{~b}$ show ice necks holding beads in place. Neck shapes are consisent with predictions of ice nucleation and growth based on surface energy. Figure 5c shows beads embedded in pore-filling ice; the cloudy appearance suggests the presence of air bubbles or other defects. Figure $5 \mathrm{~d}$ shows sockets of ice formerly occupied by beads. Figures $5 \mathrm{e}$ and $5 \mathrm{f}$ show fine necks and porefilling ice, respectively. Air bubbles trapped within the ice are indicated by arrows.

regions of our sample [e.g., Elbaum et al., 1993] they would be discontinuous or absent at greater depths with lower temperatures.

[35] Small chips of ice-encrusted beads from several sample depths were examined with an optical microscope in the cold room; Figure 5 displays selected images. Figures 5a and $5 \mathrm{~b}$ show ice necks connecting individual beads to a larger bulk mass of beads and ice. The necks form annuli around grain contacts, the edges of which are concave toward the contact (see Figure 5b). The shape and location of these necks is consistent with ice growth models based on free energy arguments [Hobbs and Mason, 1964]. Figure 5c shows turbid ice filling the interstices between several beads. Monocrystalline ice should not significantly scatter light and would appear clear; the turbidity implies the presence of crystalline defects or included air. Figure $5 \mathrm{~d}$ shows a socket formerly occupied by a bead, revealing the tight association and good thermal contact between ice and the grains of the medium.

[36] Figures 5e and $5 \mathrm{f}$ reveal bubbles within the ice which are tens of microns across. Spread throughout the sample, such voids could measurably reduce the bulk density of the ice. Though trapped atmospheric gases are common in compressed snowpack ice, their occurrence in diffusively derived ice has not been commented on previously. It thus seems that future landed missions which seek to extract information about ancient climates and atmospheres would be able to find trapped pockets of relic gases in subsurface ice even if that ice is not the result of precipitation. 

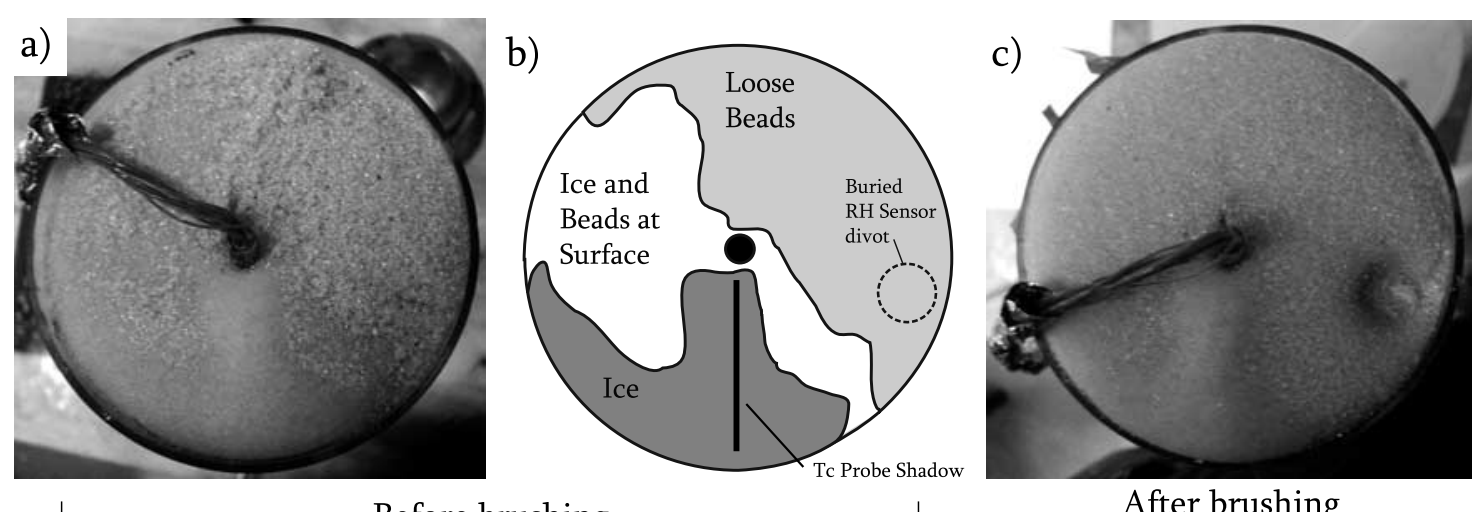

Before brushing

After brushing

Figure 6. The sample surface after $96 \mathrm{~h}$ of exposure to a humid atmosphere. (a) The surface as it appeared prior to brushing. (b) Schematic indicating a region of free surface ice, an intimate ice and bead mixture, and a region with multiple layers of ice-free beads. The position of the thermocouple wire shadow is indicated. (c) The surface after brushing away loose beads, revealing the divot formed around the warm RH/RTD sensor.

\subsection{Ice Table Morphology}

[37] Recent modeling investigations into subsurface ice deposition on Mars have shown that the ice table itself is a sharp boundary between ice-bearing and ice-free soil [Schorghofer and Aharonson, 2005]. These results differ from earlier models of Mellon and Jakosky [1993] which exhibit a more gradual transition spanning several model layers. Schorghofer and Aharonson [2005] suggest that the difference may be due to differences in numerical implementation, rather than different physics or underlying assumptions. The experiments reported here exhibit sharp interfaces, the depth and morphology of which are controlled by temperature and atmospheric vapor density. In experiments which develop ice tables beneath the sample surface the mechanical cohesion of the beads exhibits a transition between completely unconsolidated and well bound over a distance of order one grain diameter.

[38] Because of this sharpness, the ice table shape may be easily seen by brushing away the unbound beads and examining the surface of ice-cemented grains. Figure 6 shows one such example: an experiment where some surface ice developed in the colder shadow of the thermocouple wires, but where there remained some warmer, unshadowed beads not yet bound by ice. The top of the RH/RTD sensor is directly illuminated by the lamp and experiences a temperature 5-15 K warmer than the sample surface. This produces a small depression in the ice table which is $\sim 1 \mathrm{~mm}$ larger than the sensor diameter. A minor deepening of the ice table exists at the walls of the sample because of heat leakage from the caddy. The ice table is nearly flat in regions not perturbed by shadows, edge effects, or the warm sensor, with depth variations less than $1 \mathrm{~mm}$ across several $\mathrm{cm}$ of surface. Such a uniform depth of the stability boundary indicates a radially uniform temperature profile with isotherms parallel to the surface.

[39] On Mars, patches of darker or brighter dust will experience different radiative equilibrium temperatures for the same amount of insolation. Sizemore and Mellon [2006] have examined the consequences of temperature perturbations caused by this phenomenon with two- and three- dimensional numerical models. We have used our facility to experimentally examine the effects of variations in the near-surface temperature field on the ice table morphology. Glass disks $2.5 \mathrm{~mm}$ thick and $25.4 \mathrm{~mm}$ in diameter with various surface coatings are used to produce surface temperature perturbations. A gold-coated disk, a matte black disk, and an unaltered disk (control) were placed on the sample surface, as seen in Figure 7a. Samples were chilled and exposed to a humid atmosphere for $48 \mathrm{~h}$. A T-type thermocouple epoxied to the underside of each disk measured equilibrium temperatures of $263.1 \mathrm{~K}, 270.8 \mathrm{~K}$, and $268.2 \mathrm{~K}$, for the gold, black, and control disks, respectively. The clear control disk temperature is within $0.1 \mathrm{~K}$ of the glass beads $(268 \mathrm{~K})$. Following exposure, the disks were removed and the depth to the ice table was measured. A wire mesh placed above each sample defined the positions for $\sim 1100$ individual staggered grid depth measurements performed with a needle graduated at $1 \mathrm{~mm}$ intervals. These measurements were then interpolated to produce the depth maps seen in Figures $7 \mathrm{c}$ and $7 \mathrm{~d}$.

[40] To produce the ice table in Figure $7 \mathrm{~d}$, the vapor density at the sample surface was set at a nominal value of $1.6 \mathrm{~g} \mathrm{~m}^{-3}$. As can be seen from both the photograph taken with oblique illumination (Figure $7 \mathrm{~b}$ ) and the depth map (Figure 7d), the ice table exhibits significant undulations in depth on a scale comparable to the radius of the disks. The depth to the ice table around the rim of the gold-coated disk is the shallowest observed $(\sim 0.3-0.4 \mathrm{~cm})$. In the vicinity of the matte black disk, the depth to the ice table is on the order of $1 \mathrm{~cm}$ because of isotherm depression by the warmer disk. The clear disk had a smaller effect on the local environment. Morphological artifacts of higher-order are not apparent given the precision of the depth measurements and the overlapping regions of influence of the disks.

[41] An interesting effect of the gold-coated disk is the rim of shallow ice which gives way to a depression beneath the center of the disk. A possible explanation is inhibited vertical diffusion of water vapor because of the impermeable body of the disk, and the curved isotherms beneath it. Vapor moving laterally underneath the disk encounters temperatures where the vapor is saturated (an upward 



Figure 7. Experiments illustrating the effect of surface temperature on ice table development. (a) Disk placement on sample surface. (b) Ice table for high vapor density case $\left(\rho_{\text {air }}=1.6 \mathrm{~g} \mathrm{~m}^{-3}\right)$, photographed with oblique illumination from the bottom left. (c) Ice table depth map for the low vapor density case $\left(\rho_{\text {air }}=0.6 \mathrm{~g} \mathrm{~m}^{-3}\right)$. (d) Ice table depth map for high vapor density case $\left(\rho_{\text {air }}=1.6 \mathrm{~g} \mathrm{~m}^{-3}\right)$. Both experiments were exposed to a humid atmosphere for $48 \mathrm{~h}$.

deflection of the equilibrium ice table), and deposits. Vapor densities in the cold regions under the central portion of the disk are thus small and deposit a negligible amount of ice.

[42] An experiment with an identical setup and duration but less than half of the atmospheric vapor density $\left(\rho_{\text {air }}=\right.$ $0.6 \mathrm{~g} \mathrm{~m}^{-3}$ ) was performed, and the depth map is shown in Figure $7 \mathrm{c}$. The lower vapor density resulted in a slower upward migration of the ice table. After $48 \mathrm{~h}$, the mean depth is $2 \pm 0.1 \mathrm{~cm}$, compared to $0.8 \pm 0.3 \mathrm{~cm}$ for the experiment at higher humidity. At lower humidity, the ice table topography is subdued: the effect of the gold-coated disk is still seen over approximately half of the disk's surface, but the central depression effect and the discernible outline of the disk itself are absent. The greater thickness of low thermal conductivity ice-free beads insulates the ice table from the temperature perturbations at the surface. On Mars as in these experiments, the ice-free regolith above the ice table will rapidly attenuate temperature heterogeneities, reducing the sensitivity of ice-table morphology to surface albedo variations as a function of depth.

\subsection{Ice Table Depth}

[43] To explore the depth of the ice table, several experiments were run for $24 \mathrm{~h}$ with different values of atmospheric vapor density. Figure 8 shows the results of eight experiments, four run at the nominal vapor density of $1.6 \mathrm{~g} \mathrm{~m}^{-3}$, and four at reduced chamber humidities.

[44] The depth of the ice table and the total quantity of ice deposited in a given time depends sensitively on the atmospheric vapor density. The equilibrium ice table depths for the vapor densities $0.6,0.85,1.1,1.35$, and $1.6 \mathrm{~g} \mathrm{~m}^{-3}$ under a $13 \mathrm{~K} \mathrm{~cm}^{-1}$ gradient and a surface temperature of $268 \mathrm{~K}$ are, respectively, $1.4,1.1,0.92,0.75$, and $0.61 \mathrm{~cm}$. Initially, significant ice is deposited several centimeters below the equilibrium depth. Higher vapor densities allow earlier ice deposition at a given level. Once ice has deposited, local temperature gradients in the ice control vapor flux, and the deep ice becomes isolated from the atmospheric vapor content. Experiments with high value of $\rho_{\text {air }}\left(1.1-1.6 \mathrm{~g} \mathrm{~m}^{-3}\right)$ experience early deposition at depth and subsequently evolve at similar rates. Thus, the data for such experiments overlap between the base and $\sim 3 \mathrm{~cm}$ in 


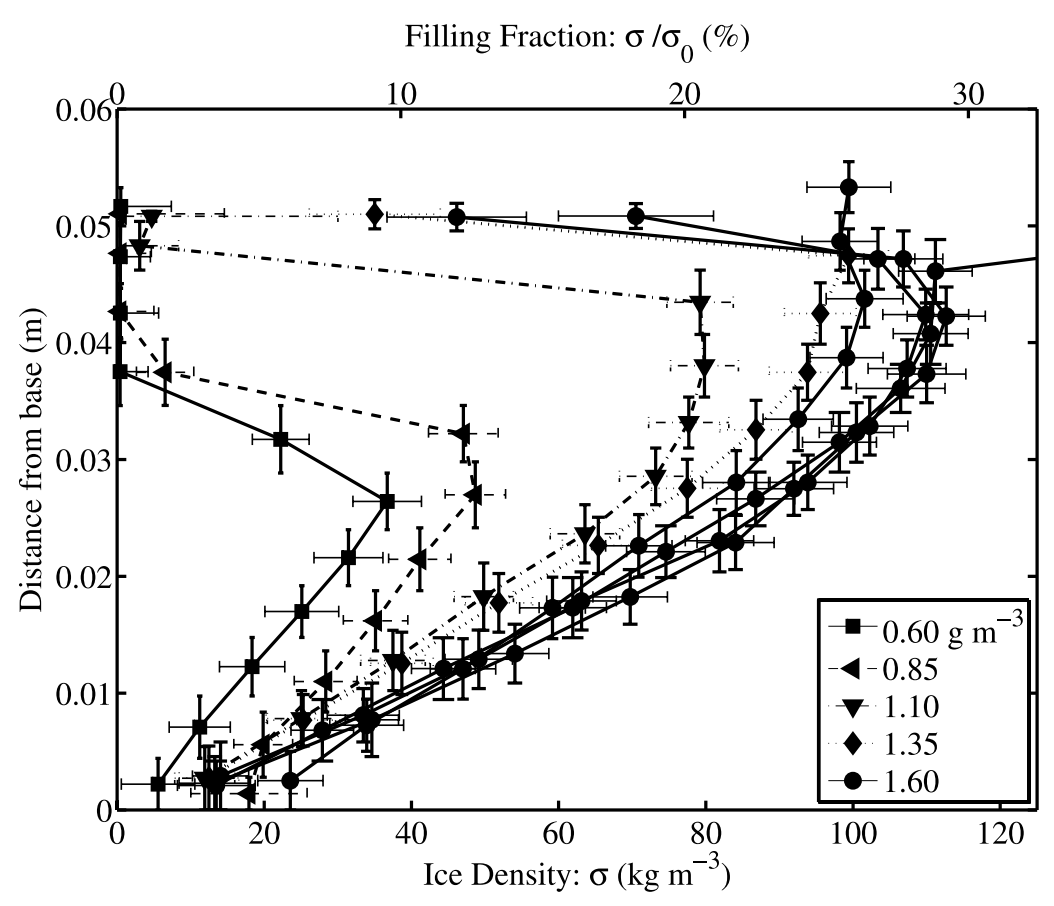

Figure 8. Ice content data for experiments run for $24 \mathrm{~h}$ at various vapor densities. Ice contents are reported as density of deposited ice with respect to total volume $(\sigma)$ and fraction of pore space filled with ice, assuming a constant ice-free porosity of $42 \%$. The maximum density of ice is thus $\phi_{0} \rho_{\text {ice }}=\sigma_{0}=$ $385.6 \mathrm{~kg} \mathrm{~m}^{-3}$. The depth to the ice table in each experiment is included in Table 1 .

Figure 8 . The phenomenology of the early transients in ice deposition is examined further in section 5.4.

\subsection{Ice Profile}

[45] The structure of the ice distribution beneath the ice table, in a region which may be termed the cryosphere, is revealed in these experiments as a function of both depth and time and is interpretable in terms of the experimental conditions and durations. The water contents determined from gravimetric analysis reveal profiles of ice content which decrease monotonically below the depth of maximum filling. Low humidity or short-duration experiments give rise to an ice table beneath an ice-free layer, while other conditions (e.g., longer durations, higher vapor densities) produce stable surface ice. Figure 9 plots the final ice content results of experiments run for different periods of exposure to an atmosphere with a surface vapor density (reported by sensor 1) of $1.6 \mathrm{~g} \mathrm{~m}^{-3}$. As described in section 4.2 , the actual vapor density driving the flux of water is believed to be somewhat higher. The maximum filling fraction observed, approximately $90 \%$, was observed at the end of the longest-duration experiment.

[46] As revealed in Figure 9, the ice contents first increase rapidly, followed by a reduced rate of ice accumulation. The shortest experiments were performed in additive 6-h increments, the second set's durations were $24 \mathrm{~h}$ apart, and the final long-duration experiments are $110 \mathrm{~h}$ and $199 \mathrm{~h}$ longer than the next shorter runs.

\subsection{Ice Deposition Rate}

[47] As described in the previous section, the rate of ice growth throughout the column is not uniform where ice is present, because the third derivative of vapor pressure with depth does not vanish. Initially, ice grows most quickly at the top of the ice table where the vapor density gradient is steepest. Beneath the ice table, the rate of ice growth also varies with time. This may be seen in the spacing of the contours in Figure 9, but is more clearly evident in Figure 10, where the difference in ice contents between successively timed experiments are divided by the intervals between those experiments, thus plotting the incremental change in ice content, $\delta \sigma / \delta t$. Similarly timed experiments are differenced from an experiment of significantly shorter $(>5 \mathrm{~h})$ duration.

[48] Figure 10 reveals the depth of greatest ice growth in a given time increment. For the first $6 \mathrm{~h}$ (dark red), most ice deposits between $1.5-2.5 \mathrm{~cm}$ beneath the surface. In the next longer increment, from 6-12 h (bright red), the greatest growth is at about $1.0 \mathrm{~cm}$ depth. Between $18 \mathrm{~h}$ to $48 \mathrm{~h}$ (orange and yellow), the greatest ice deposition occurs in the uppermost soil layer. At all subsequent times, the ice growth progresses more uniformly with depth, but accumulation rates decrease as exposure time to a humid atmosphere continues to increase.

[49] In a porous regolith, the free gas diffusion coefficient is reduced by a factor proportional to the cross-sectional area of the medium. An equivalent diffusivity, $D$, may be written in a regime which involves both molecule-molecule collisions and collisions with the pore walls to a significant degree. This is given by the Bosanquet equation,

$$
\frac{1}{D}=\frac{1}{D_{F}}+\frac{1}{D_{K}}
$$

where $D_{F}$ is the Fickian diffusivity, which dominates when collisions between gas molecules dominate the behavior of 


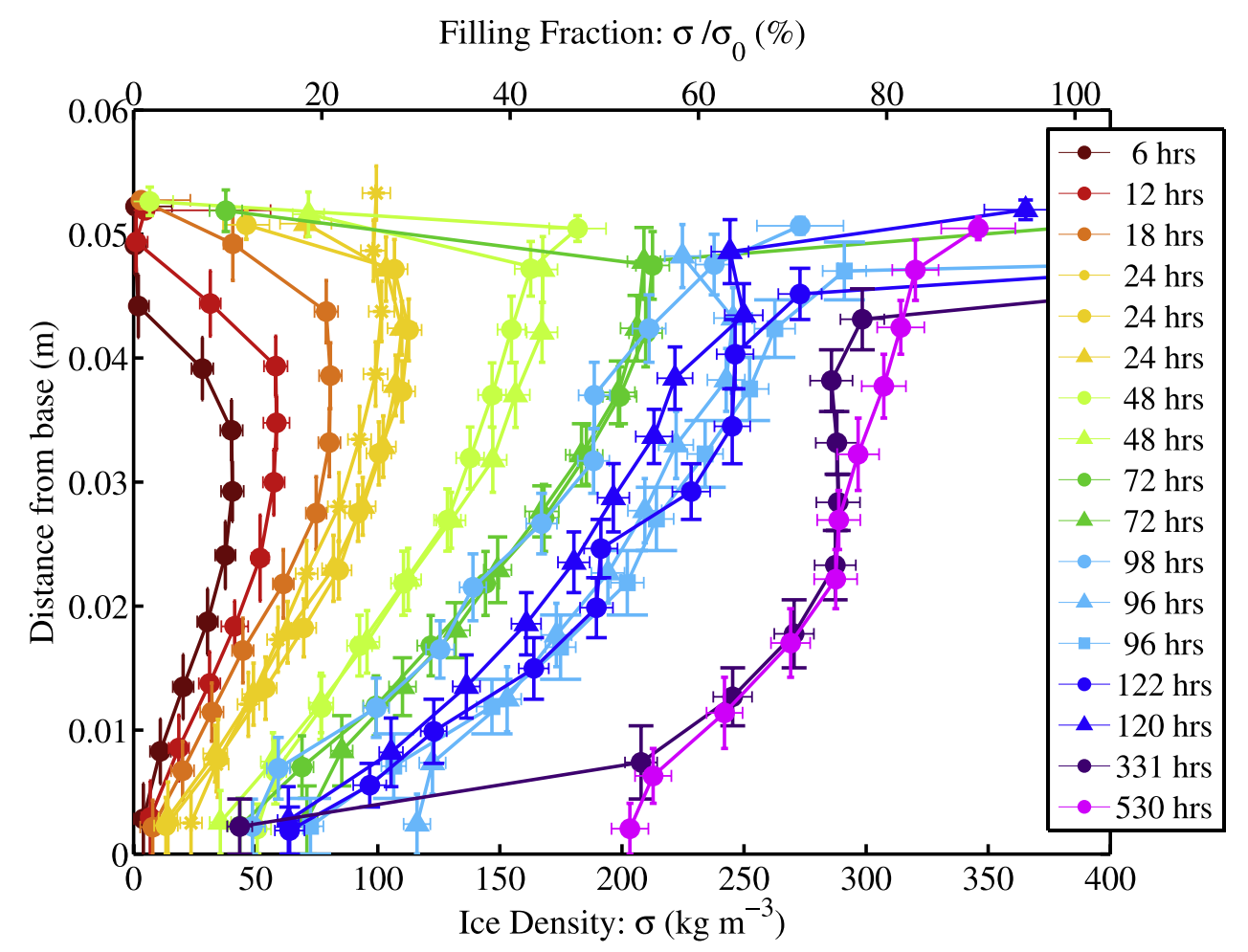

Figure 9. Ice content data for experiments run at a nominal surface vapor density of $1.6 \mathrm{~g} \mathrm{~m}^{-3}$ and various durations. After $\sim 48 \mathrm{~h}$, free ice deposits at the surface and forces the uppermost point for some samples to have a measured ice content off scale to the right. The colors correspond to duration of exposure to a wet atmosphere, as indicated by the legend. Experiments with similar durations are given the same color but different symbols.

the gas movement, and the Knudsen diffusivity $D_{K}$ which dominates at low pressures when molecules collide more frequently with pore walls than with each other [Pollard and Present, 1948]. As ice accumulates, the pore space geometry changes, altering the porosity and tortuosity, and increasing the frequency of molecule-wall collisions as the pore size is reduced. $D_{F}$ and $D_{K}$ depend upon the pore geometry in potentially different ways, which may also be different from the behavior in a dry medium with the same geometry. These differences arise both because of the simultaneous source and sink nature of icy walls and the strict dependence of the vapor density on temperature in saturated pore gas. These effects are examined further in section 5.2.

\section{Numerical Model and Experiments}

\subsection{Description of Ice-Filling Model}

[50] Vapor transport and phase transitions are calculated with the numerical code described by Schorghofer and Aharonson [2005], which we use to model the experiments presented here. Fick's law, local mass conservation, and the ideal gas law lead to

$$
\frac{\partial}{\partial t}\left[\phi_{0}\left(1-\frac{\sigma}{\sigma_{0}}\right) \frac{p_{1}}{T}+\frac{R}{18} \sigma\right]=\frac{\partial}{\partial z}\left[D(\phi, \tau) \frac{\partial}{\partial z} \frac{p_{1}}{T}\right],
$$

where $p_{1}$ is the partial pressure of $\mathrm{H}_{2} \mathrm{O}$. Within the icecontaining region the ice growth can also be obtained from equations (2) and (3), but these equations do not directly provide the vapor density in a dry layer or the depth of the ice interface. Hence, we use the partial differential equation (5), which includes these effects.

[51] Equation (5) is numerically solved with an explicit forward difference scheme, with a spatial resolution of $0.5 \mathrm{~cm}$, the spacing of the temperature sensors in the experiment. Models may also be run with temperatures interpolated to a finer grid spacing. Experimentally measured temperature profiles and surface vapor densities are used as input. To remove artificial temperature inflections caused by thermocouple misalignment from exact halfcentimeter spacing, the temperature data are fit with a fourth-degree polynomial prior to being used as input. Small time steps are required for numerical stability, and the program subdivides measured temperature profiles, typically available in 1-min intervals, into smaller time steps of $50-1000 \mu$ s by linear interpolation.

\subsection{Comparison to Models and Uncertainties}

[52] In this section we consider five quantities which impact the agreement of the model with the experimental ice contents. They are: headspace vapor density $\left(\rho_{\text {air }}\right)$, icefree diffusion coefficient $\left(D_{0}\right)$, temperature profile, density of deposited ice $\left(\rho_{\text {ice }}\right)$, and what we term the constriction exponent $(n)$.

[53] Experiments performed with three humidity sensors placed as indicated in Figure 3 recorded vapor densities consistently $80 \%$ higher at sensors 2 and 3 than at sensor 1 . The possibility of a malfunction in sensor 1 was examined and discarded by swapping the sensors positions during one 


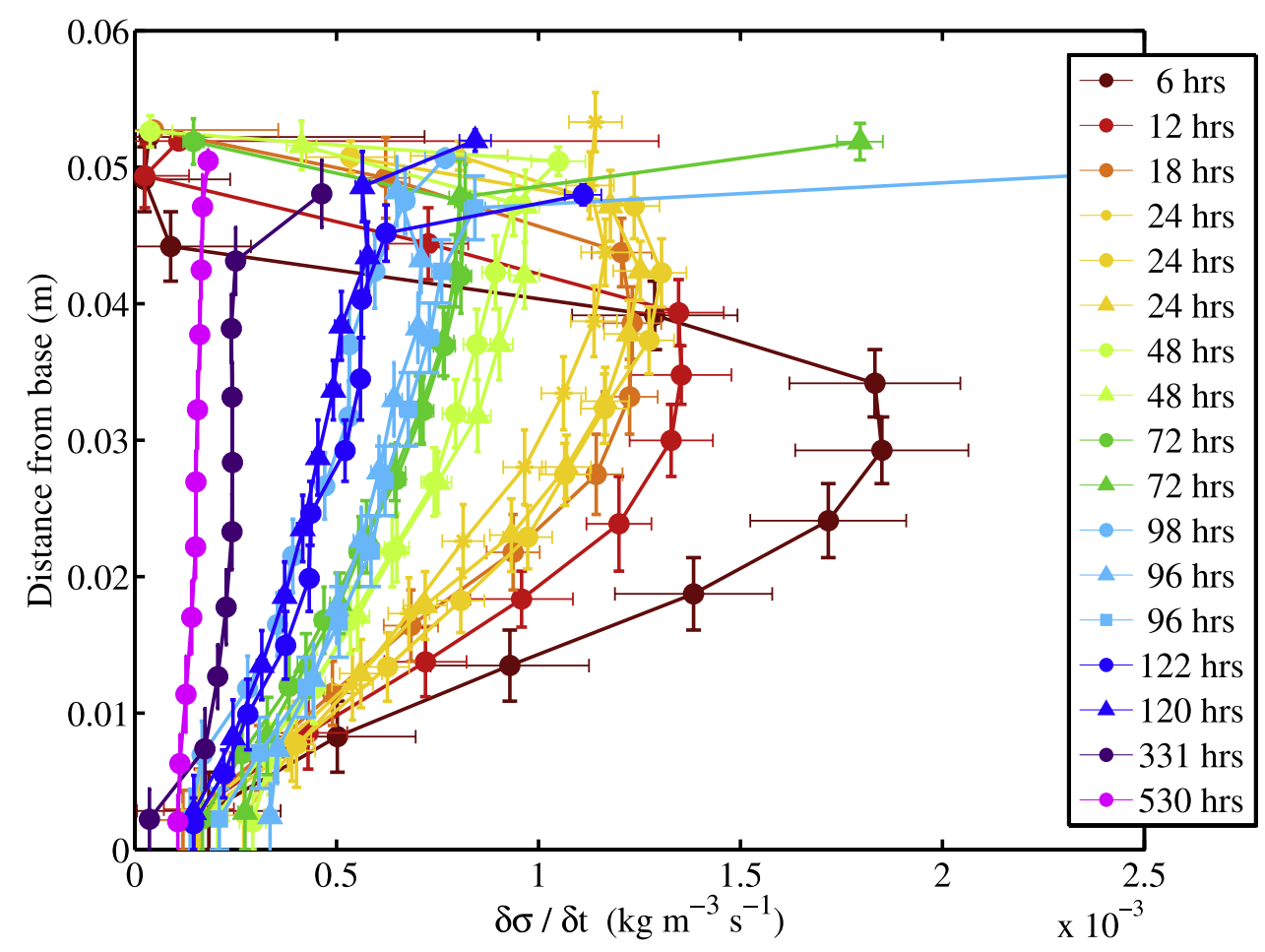

Figure 10. The change in ice content for a given depth divided by the time interval for growth. The legend (distinct from Figure 9) indicates the end of the time interval (e.g., " $6 \mathrm{~h}$ " is $0-6 \mathrm{~h}$, " $48 \mathrm{~h}$ " is $24-$ $48 \mathrm{~h}$, etc.). The most rapid ice growth occurs during the earliest times of exposure $(0-6 \mathrm{~h})$, and the maximum can be seen to evolve from a depth $\sim 2.0 \mathrm{~cm}$ for $0-6 \mathrm{~h}$ to $\sim 1.0 \mathrm{~cm}$ for $6-12 \mathrm{~h}$, to the surface for times between 12 and $24 \mathrm{~h}$. Subsequent to this, the rates are much smaller, are much more uniform across the sample depth, and continue to decrease with time.

experiment and observing the same trend of humidities as a function of height from the sample surface. Additionally, ice was observed to deposit at the sample surface after $\sim 48 \mathrm{~h}$ while the vapor density reported by sensor 1 implied an ice table $\sim 0.6 \mathrm{~cm}$ beneath the surface. We therefore surmise that (1) the atmosphere above the sample in these experiments is not well mixed and (2) the bulk atmospheric vapor density driving the flux of water vapor is higher than $1.6 \mathrm{~g}$ $\mathrm{cm}^{-3}$ by almost a factor of 2 . Compensation equations for sensor temperature are provided by the vendor; however the range of validity for those expressions may not extend to low-pressure environments.

[54] This uncertainty in $\rho_{\text {air }}$ affects estimates of ice table depth and ice contents in experiments of less than $\sim 48 \mathrm{~h}$ duration. In Figure 11, the ice content measured at the end of a $24 \mathrm{~h}$ experiment is displayed along with results of numerical simulations (section 4.1). Model runs use the experimentally measured subsurface temperature profile. When vapor densities reported by sensor 1 are used, the model output (Figure 11, triangular symbols) underpredicts ice contents relative to the experimental data (filled circles, solid black curve) in the upper half of the sample. When, instead, vapor densities are prescribed constant values greater than those reported by sensor 1, the models develop quantities of ice at shallower depths similar to that in the experimental profile. The density giving the best match between models and experiments varies from $\rho_{\text {air }}=2.0$ $3.2 \mathrm{~g} \mathrm{~m}^{-3}$, with lower values producing the best correspon- dence with the shortest times. For $48 \mathrm{~h}$ experiments, good agreement between experiments and models is obtained for surface vapor densities of $\sim 3.2 \mathrm{~g} \mathrm{~m}^{-3}$. This value is equal to the vapor density of ice at $268 \mathrm{~K}$ and $600 \mathrm{~Pa}$, which are the conditions maintained at the surface of the sample. This supports the conclusion that free surface ice becomes the dominant vapor source at times beyond $\sim 48 \mathrm{~h}$.

[55] An important feature of Figure 11 is that all model runs, despite differing surface vapor densities, agree beneath the depth where ice is present; the curves merge onto a single line. Flux beneath the ice table is governed by the local vapor pressure, which is controlled by temperature and is independent of the atmospheric vapor density. The important differentiation among models with different surface vapor densities is the time when a particular depth begins to deposit ice. Higher vapor densities advance this point to earlier times at a given depth.

[56] In the rest of this section dealing with model and experiment agreement, the focus is primarily on runs of $48 \mathrm{~h}$ or longer, and the discussion is therefore specific to experimental data and models run with surface vapor densities of $3.2 \mathrm{~g} \mathrm{~m}^{-3}$. When such models are run, they consistently overpredict the abundance of ice in the sample, indicating that some other factor besides vapor density is acting to reduce ice deposition.

[57] Measurements of $D_{0}$ for the $500 \mu \mathrm{m}$ glass beads have an uncertainty of about $10 \%$ (section 2.4). Additional model runs were performed using $D_{0}=4.2 \mathrm{~cm}^{2} \mathrm{~s}^{-1}$ to determine if 


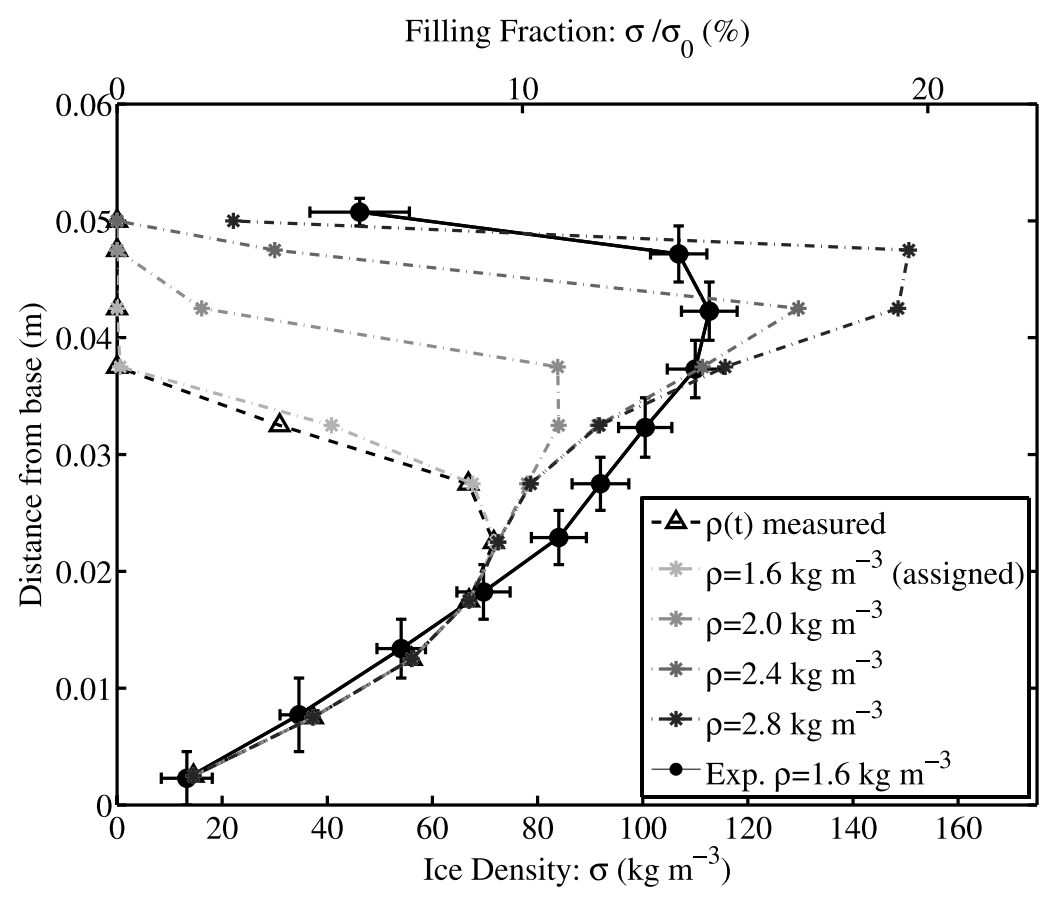

Figure 11. Ice content data for a 24-h exposure experiment at a nominal measured vapor density of $1.6 \mathrm{~g} \mathrm{~m}^{-3}$ (black circles, solid line), compared to model simulations (broken lines) using experimental temperature data interpolated to $2 \times$ resolution. One model was run with surface vapor densities as measured (triangles), and several were run with assigned values of $\rho \equiv \rho_{\text {air }}$ (asterisks). Notice that the models agree below the depth where ice has accumulated, indicating gradient control by the saturation vapor pressure.

reasonably lower values of $D_{0}$ would bring closer agreement with experimental results. Such models do exhibit reduced ice contents, but do not bring the model output into good agreement with the data. We therefore conclude that the major source of variation lies elsewhere and use $D_{0}=$ $4.7 \mathrm{~cm}^{2} \mathrm{~s}^{-1}$ for subsequent model analyses.

[58] A $\sim 1 \mathrm{~mm}$ vertical deviation in thermocouple placement results in $\sim 1 \mathrm{~K}$ temperature offset, corresponding to a $10 \%$ variation in vapor density at the highest $(\sim 268 \mathrm{~K})$ experiment temperatures. Models run with imposed $1 \mathrm{~K}$ shifts to lower temperatures produced insignificant differences in ice abundances compared to unshifted temperature data.

[59] The maximum observed filling fraction is less than $100 \%$, suggesting the possibility that the deposited ice is less dense than pure ice. This is consistent with micrograph images (see Figure 5) which show encapsulated bubbles and turbid ice. If the numerical model is modified such that the density of deposited ice is lower, the results can be made to match more closely with the experimental data. Figure 12 shows such results for four selected data sets and their attendant model runs using ice densities of 918 and $734 \mathrm{~kg}$ $\mathrm{m}^{-3}$, the latter being equivalent to $80 \%$ maximum filling. Such a reduced density improves the model fit, but filling fractions up to $90 \%$ are observed in the longest experiment. In the simplest case we would assume that all deposited ice has the same density, which should be at least the maximum observed in any experiment, suggesting that a density of $734 \mathrm{~kg} \mathrm{~m}^{-3}$ is too low. It is possible that the density of deposited ice varies depending on local geometric effects, temperature, and time of deposition. We do not measure the density of the deposited ice directly and thus cannot constrain ice density as a function of time. Given this uncertainty and our observation of $90 \%$ filling (implied ice density $\sim 826 \mathrm{~kg} \mathrm{~m}^{-3}$ ), we consider another possibility: a nonlinear dependence of flux on porosity and filling fraction, which may also be termed pore space constriction.

[60] The model results presented thus far have used a linear relation between diffusivity and filling fraction, as used by Mellon and Jakosky [1993], to account for a changing rate of ice deposition. Constriction of an ice-free medium, by a process which reduces the porosity and increases the tortuosity, would result in reduced values of $D$ as the pore volume was diminished. But in an ice-rich medium, the concept of "constriction" becomes less clear since the gradient driving diffusion at any point does not depend on the geometry of the medium. Nevertheless, a reduction in deposition rate with increased filling fraction is observed and so is parameterized as a constriction phenomenon. In this scheme, the porosity at time $t$ is

$$
\phi(t)=\phi_{0}\left(1-\frac{\sigma(t)}{\sigma_{0}}\right) .
$$

The ice-free diffusion coefficient $D_{0}=D\left(\phi_{0}\right)$ is thereby modified by a factor of $\phi / \phi_{0}$. The model allows that the ice accumulation rate changes as a result of changing values of D. The model of Mellon and Jakosky [1993] subsumes all variation in pore geometry into the value of the porosity, $\phi$, 


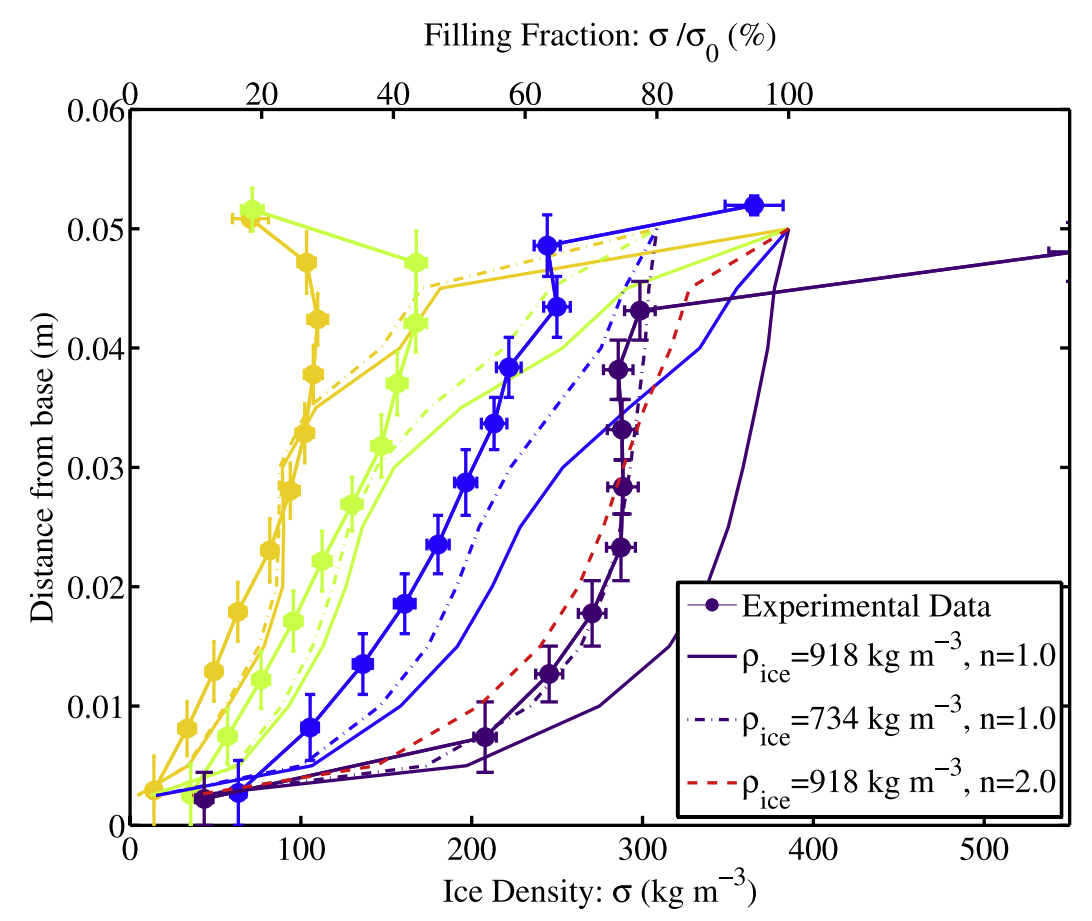

Figure 12. Experimental data and model outputs for 24, 48, 120, and 330 h experiments. Experimental data are filled circles with error bars. Model results vary as the maximum density of deposited ice is changed, and the best agreement for the experiments with high filling fractions appears to be with $\rho_{\text {ice }}=$ $734 \mathrm{~kg} \mathrm{~m}^{-3}$, or $80 \%$ the density of pure ice. For times shorter than $\sim 48 \mathrm{~h}$, models which assume diffusivity is linearly proportional to filling fraction agree with the experimental data. A single dashed line (in red) indicates the model output for the $330 \mathrm{~h}$ experiment if $100 \%$ ice density is assumed, and diffusivity varies as the square of the filling fraction (i.e., constriction exponent equals 2).

and leaves the tortuosity term unaltered. This was justified because of a lack of experimental or theoretical data on the effects of deposited ice on tortuosity.

[61] We find, however, that agreement between our experimental data and the ice contents predicted by our models can be improved if the density is maintained at $\sim 918 \mathrm{~kg} \mathrm{~m}^{-3}$, but the constriction factor is modified by an exponent, $n$ :

$$
D=D_{0}\left(1-\frac{\sigma}{\sigma_{0}}\right)^{n}
$$

[62] The reduction of porosity (since the area through which vapor can pass integrated over depth is the void volume) due to deposited ice is linear in $\sigma$, hence our base model and that of Mellon and Jakosky [1993] assume $n=1$. It can be seen in Figure 12 that models with $n=1$ match the data well for filling fractions of $\sim 40 \%$ or less, but that they overpredict the amount of ice deposited for longer times with higher filling fractions. A model run for the $330 \mathrm{~h}$ experiment, wherein filling fractions are $>70 \%$, which uses $n=2$ and $\rho_{\text {ice }}=918 \mathrm{~kg} \mathrm{~m}^{-3}$ is plotted in Figure 12 as a red dashed curve and is seen to be in close agreement with the experimentally measured ice contents. The degree of agreement between this model and the experimental data is comparable to that between the data and the model with $n=1$ and $80 \%$ ice density, but this scenario is not favored because observed densities reach at least 90\%. Changes other than reduction of porosity, e.g., the shortening of molecular hop distances as ice reduces the available volume, add additional effects which cause a nonlinear dependence of $D$ on $\sigma$ when filling fractions are large. In our model parameterization, these effects are captured in $n$, as described in section 5.2.

\subsection{Time-Varying Diffusion Coefficients}

[63] The reduction in $\partial \sigma / \partial t$ with time is mainly attributable to changes in $D$ since $\partial \rho_{1} / \partial z$ is approximately constant in time. The diffusivity as a function of time can be extracted from the available experimental data by making the simplifying assumption that variations of $D$ with depth are slow. The right hand side of equation (2) may then be used to estimate the diffusion coefficient as a function of ice density. The relation used is:

$$
\frac{\sigma(t+\delta t)-\sigma(t)}{\delta t}=D\left(t+\frac{\delta t}{2}\right) \frac{1}{\delta t} \int_{t}^{t+\delta t} \frac{\partial^{2} \rho_{1}}{\partial z^{2}} d t^{\prime}
$$

where $\delta t$ indicates the time interval separating successive experiments. The accumulated quantity of ice in an interval, $\delta \sigma / \delta t$, divided by average values of the density divergence in the same interval gives a proxy for $D$, which may be plotted versus the average ice content over $\delta t$ at a particular depth. Figure 13 thus displays the change in diffusivity due 


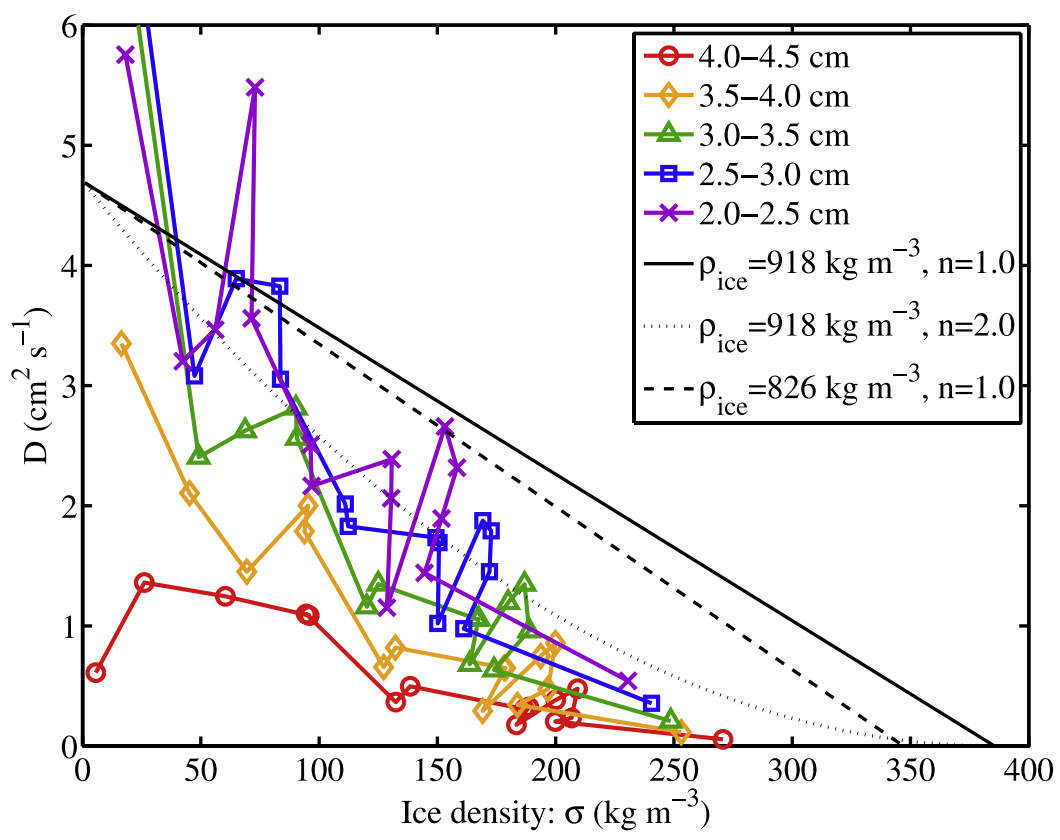

Figure 13. Plot of the diffusion coefficient evaluated using the technique described in section 4.3 versus ice content for five deep levels in the sample column. Overplotted are expected variations in $D$ with $\sigma$ using $\rho_{\text {ice }}=918 \mathrm{~kg} \mathrm{~m}^{-3}$ for $n=1$ (solid) and $n=2$ (dotted). The trend in the diffusion coefficient exhibits a concave-upward slope which is consistent with $n$ greater than unity. If the density of deposited ice were $90 \%$ of pure ice density, and $n=1$, the diffusion coefficient would follow the dashed line.

to ice deposition in the deepest layers where the changes in ice content are easiest to observe.

[64] Systematic differences among experiments result in a considerable scatter in Figure 13, but an overall trend to lower values of $D$ can be discerned. The trends are consistent with the expected variation of $D$ with $\sigma$ for a constriction exponent greater than unity $(n=1$ and 2 are plotted as solid and dotted lines, respectively). If we constrain $n$ to be equal to unity, but allow the maximum ice density relative to total volume to be less than $386 \mathrm{~kg} \mathrm{~m}^{-3}$, then the linear trend (dashed line) would reach a smaller maximum value. But filling fractions of $90 \%$ imply a $\sigma$ of at least $347 \mathrm{~kg}$ $\mathrm{m}^{-3}$. The trend of $D$ with $\sigma$ using this lower density and $n=$ 1 does not match the data as well as the data with a higher constriction exponent, suggesting that $D(\sigma)$ depends on $\sigma$ and is nonlinear for high filling fractions.

[65] The reduced deposition rate as a function of time exhibited by these data is consistent with a constriction-like behavior as employed by Mellon and Jakosky [1993]. The close agreement between the reduced rate of ice accumulation in models and experimental data improves confidence that the model employing nonlinear adjustment of $D$ with $\sigma$ more closely captures the physics of the ice deposition process. Both effects of reduced ice density and nonlinear dependence of deposition rate on filling fraction may be important in controlling the evolution of ice at high filling fractions obtained for long exposures to ice growth conditions.

\subsection{Long-Duration Behavior}

[66] The longest experiment was run for $530 \mathrm{~h}$ of exposure to humid gas. The rate of change between the 330 and $530 \mathrm{~h}$ experiments is very small, indicating significant reduction of ice deposition rates in the shallow layers and concurrent reduction of these rates at greater depths. The numerical model has been run for $6000 \mathrm{~h}$ of experiment time using a static, linear temperature profile of the same magnitude as used in the experiments. The other parameters are a constriction exponent of $n=2$, an ice density of $918 \mathrm{~kg}$ $\mathrm{m}^{-3}$, the total pressure of $600 \mathrm{~Pa}$, and surface densities of 1.6 and $3.2 \mathrm{~g} \mathrm{~m}^{-3}$.

[67] Figure 14 plots the cumulative ice content as a function of time for the two model runs (broken lines), column-integrated ice contents for the experiments (filled symbols), and the local column ice density for the most icerich layer in each experiment, scaled by a factor of 10 (open symbols). The column integrated ice contents are computed over the depth of the ice-bearing sample. Both models are seen to converge asymptotically to the maximum theoretical ice content, $\sigma_{0} / Z$, with the difference being $<5 \%$ after $\sim 5000 \mathrm{~h}$. The experimental data agrees well with the model using the higher vapor density, particularly for times greater than $24 \mathrm{~h}$, corresponding to surface ice at $268 \mathrm{~K}$, rather than the atmosphere, being the dominant vapor source.

[68] The experimental data are consistent with the asymptotic approach of the ice content to total filling. Further experiments which achieve high filling fractions may improve understanding of the ice deposition process, potentially revealing important phenomena that only affect ice deposition when pore filling fractions are high.

\section{Discussion}

[69] This section explores the mechanisms and implications of constrictive modification of diffusion in the context 


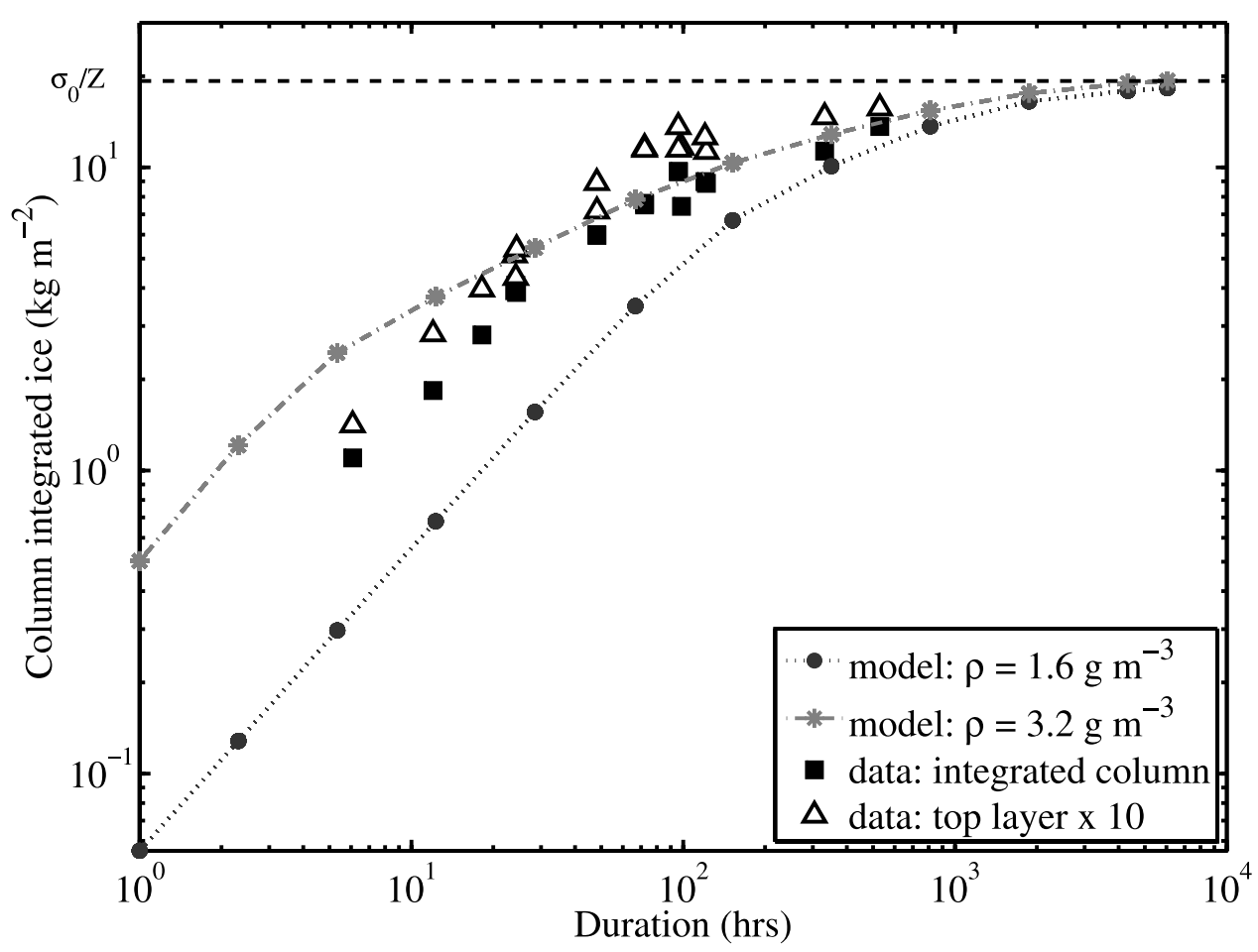

Figure 14. Column-integrated ice abundances for experiments and two long-duration models (parameters given in text). Solid symbols are integrated column densities, and open symbols represent ice contents in the uppermost ice-bearing layer (i.e., the most ice-rich layer) scaled by a factor of 10 . Model runs with vapor densities of $3.2 \mathrm{~g} \mathrm{~cm}^{-3}$ match total ice abundances well after $24 \mathrm{~h}$, supporting the conclusion that the driving vapor density is that of the surface ice at $268 \mathrm{~K}$. Maximum column density for ice in a $42 \%$ porosity medium would be $\sigma_{0} / Z=19.3 \mathrm{~kg} \mathrm{~m}^{-2}$.

both of the experiments reported and for the martian regolith.

\subsection{Theoretical Uncertainties in Vapor Transport}

[70] The governing equation for the diffusive transport of water vapor (denoted by subscript 1 ) in $\mathrm{CO}_{2}$ (denoted by subscript 2) is given by [Landau and Lifshitz, 1987]

$$
J_{1}=-\rho_{0}\left[D_{12} \frac{\partial}{\partial z} \frac{\rho_{1}}{\rho_{0}}+\frac{D_{T}}{T} \frac{\partial T}{\partial z}\right]
$$

where $J_{1}$ is the diffusive mass flux of gas $1, \rho_{0}$ is the total mass density, $\rho_{0}=\rho_{1}+\rho_{2}, D_{12}$ the mutual diffusion coefficient of $\mathrm{H}_{2} \mathrm{O}$ in $\mathrm{CO}_{2}$ in the absence of a porous medium. $D_{T}$ is the coefficient for "thermodiffusion," and $T$ the temperature. We neglect the contribution of barodiffusion to the vapor flux.

[71] In Hudson et al. [2007] we present a review of the thermal diffusion effect and show that it is a negligible contributor to the vapor flux in the experiments described therein. Here, we revisit the issue for the high thermal gradients in the present experimental setup.

[72] The concentration-dependent thermal diffusion factor $\alpha_{T}$ is at most $\alpha_{T} \approx 0.8$ for $\mathrm{H}_{2} \mathrm{O}-\mathrm{CO}_{2}$, using the conservative elastic sphere model [Chapman and Cowling, 1970]. By definition $D_{T} / D_{12}=\alpha_{T}\left(n_{1} / n_{0}\right)\left(n_{2} / n_{0}\right)$, where $n$ indicates number densities, and $n_{0}$ is the total number density of both species. At low concentration, $D_{T} / D_{12} \approx$
$\alpha_{T}\left(\rho_{1} / \rho_{0}\right)\left(\mu_{2} / \mu_{1}\right)$, where $\mu_{1}$ and $\mu_{2}$ are the masses of $\mathrm{H}_{2} \mathrm{O}$ and $\mathrm{CO}_{2}$ molecules, respectively.

[73] In steady state, the net $\mathrm{H}_{2} \mathrm{O}$ flux vanishes, rendering the ratio of thermodiffusion flux to concentration driven flux as an inappropriate measure. Instead, we estimate what the change in ice table depth $\delta z$ due to thermodiffusion in our laboratory setup is if the concentration were small, which is determined by

$$
-\rho_{0} D_{T} \frac{1}{T} \frac{\partial T}{\partial z}=\frac{\partial J_{1}}{\partial z} \delta z
$$

The total diffusive flux, $J_{1}$, is approximately, $-D_{12} \partial \rho_{1} / \partial z$, and thus

$$
\rho_{1} \alpha_{T} \frac{\mu_{2}}{\mu_{1}} \frac{1}{T} \frac{\partial T}{\partial z}=\frac{\partial^{2} \rho_{1}}{\partial z^{2}} \delta z
$$

From equation (3) and $H /(R T) \approx 23 \gg 1$, we obtain an approximate expression for $\partial^{2} \rho_{1} / \partial z^{2}$ which we then use in equation (11) to obtain,

$$
\alpha_{T} \frac{\mu_{2}}{\mu_{1}} \approx \delta z\left(\frac{H}{R T}\right)^{2} \frac{1}{T} \frac{\partial T}{\partial z} .
$$

Hence, $\delta z \lesssim 8 \times 10^{-4} \mathrm{~m}$, which is negligible compared with the relevant length scales. 
[74] The contribution of advection or bulk flow to the vapor transport is also small. The sample container has an impermeable bottom boundary, such that bulk motion can only be caused by the displacement of gas from the growing ice volume, surely negligible, and the contribution of the $\mathrm{H}_{2} \mathrm{O}$ flux to the overall motion. The latter should be on the order of $p_{1} / p_{0}$; the maximum $p_{1}$ is reached at the ice table, where $p_{s v}(268 \mathrm{~K})=397 \mathrm{~Pa}$, while $p_{0}=600 \mathrm{~Pa}$. Figure 1 demonstrates that rapid ice growth only occurs when the equilibrium vapor pressure of $\mathrm{H}_{2} \mathrm{O}$ is not much smaller than the total pressure.

[75] By eliminating the advective velocity from equations for the total flux of interdiffusing species, we find that the inward flux is larger than what we obtain from diffusion alone by a factor of $\rho_{0} /\left(\rho_{0}-\rho_{1}\right)$ [Landau and Lifshitz, 1987; Hudson et al., 2007]. This expression is not applicable for Knudsen diffusion, but qualitatively indicates that advection increases the flux in our experiments. For model runs greater than $48 \mathrm{~h}$, the excess ice relative to observations using a linear dependence of $D$ on filling fraction, i.e., $n=1$, can therefore not be explained by an unaccounted advective contribution.

\subsection{Constriction Physics}

[76] This section explores the mechanisms and implications of constrictive modification of diffusion in the context both of the experiments reported and for the martian regolith. In equation (7), we introduce an exponent, $n$, to modify the change in diffusion coefficient as a function of filling fraction. Theoretical investigations [Hobbs and Mason, 1964] point to preferential deposition of ice at high negative curvature surfaces such as grain contact points. The micrograph observations of samples at the conclusion of experiments are consistent with this growth habit. A changing geometry of the pore space during ice deposition results in a nonlinear dependence of $D$ on $\sigma$ at high filling fractions. A cause of this phenomenon arises from reduced pore space volume, which results in a greater frequency of molecule-wall collisions for a given pressure. The effect of mean free path and molecular hop length on $D$ can be seen in the following derivation.

[77] The free gas diffusivity may be approximately written as

$$
D_{12}=\frac{1}{3} \bar{v}_{1} \lambda
$$

where $\bar{v}_{1}$ is the mean thermal speed of water molecules and $\lambda$ is the mean free path. The Fickian diffusion coefficient in a porous medium may be written as

$$
D_{F}=\frac{\phi}{\tau} D_{12}
$$

and the Knudsen diffusion coefficient may be written as

$$
D_{K}=\frac{1}{3} \bar{v}_{1} l \frac{\phi}{\tau},
$$

where $l$ is a measure of characteristic hop length, and any prefactor is subsumed into the relation between $l$ and pore size. This treatment is similar to the treatment of Evans et al.
[1961], who derive a Knudsen diffusion expression which is also proportional to the obstruction factor, $\phi / \tau$. Since $l$ is proportional to available pore size,

$$
l=l_{0} \phi / \phi_{0}
$$

where $l_{0}$ is for the dry porous medium. Equations (4), (13), (14), and (15) lead to

$$
D=\frac{\phi}{\tau} \frac{\bar{v}_{1}}{3}\left(\frac{1}{\lambda}+\frac{1}{l}\right)^{-1}
$$

Hence, changes in $D$ with ice content can be attributed to three contributions: porosity $\phi$, tortuosity $\tau$, and restriction in hop length $l$. If $\tau$ were constant with time, then $D$ varies proportionally with $\phi$ when $\lambda \ll l$, and it varies as $\phi^{2}$ for $\lambda$ $\gg l$. The ice deposition will simultaneously change the porosity, the pore diameter distribution, and the tortuosity. Thus, the transition to a more Knudsen-dominated regime will affect the rate of flux in addition to the effects of a changing obstruction factor.

[78] The diffusivity therefore changes from linear in $\sigma$ to a stronger dependence as deposition progresses. At high filling fractions, the $l$ term in equation (17) dominates over the $\lambda$ term, the porosity is small, and the tortuosity will become large as diffusive paths are closed off. Figure 12 shows that models with low filling fractions agree well with experimental data if the relationship between $D$ and $\sigma$ is linear, i.e., the constriction exponent $n$ equals 1 , while models for long-term experiments with high filling fraction overpredict the ice abundance with $n=1$. Equation (17) implies that the functional relationship between $D$ and $\sigma$ changes with $\sigma$, and a real system would not be described by a constriction linear in $\sigma$, as was employed in our model (section 4.3). Further study of the constriction phenomenon may reveal the appropriate prefactors and dependencies to model this transition.

\subsection{Initial Ice Deposition}

[79] The preferential deposition at grain contact points, as revealed by the micrographs in Figure 5, could first fill the smallest pore spaces and narrowest gaps between grains. Most of the flux in a medium of distributed pore sizes is carried in the largest pores [Clifford and Hillel, 1983, 1986], and this initial deposition should not greatly alter the effective diffusion coefficient. But this small amount of ice may still affect the availability of pathways to conduct vapor, changing the tortuosity of the medium.

[80] Another consequence of ice deposition is a change in bulk thermal conductivity [Paige, 1992]. The thermal conductivity of ice-free $500 \mu \mathrm{m}$ glass beads used in these experiments is $0.18 \mathrm{~W} \mathrm{~m}^{-1} \mathrm{~K}^{-1}$ [Ham and Benson, 2004]. This value is higher than the values of bulk thermal conductivity for particulate material used by Mellon and Jakosky [1995] $\left(0.02 \mathrm{~W} \mathrm{~m}^{-1} \mathrm{~K}^{-1}\right)$. Ice, on the other hand, has a bulk thermal conductivity of $\sim 2.2 \mathrm{~W} \mathrm{~m}^{-1} \mathrm{~K}^{-1}$. The addition of ice at grain contact points greatly increases the conductivity of the sample through increased area of heat conduction and the high thermal connectivity of the added material. The effect of included ice on the bulk conductivity of an ice-bearing soil was represented by Mellon et al. 


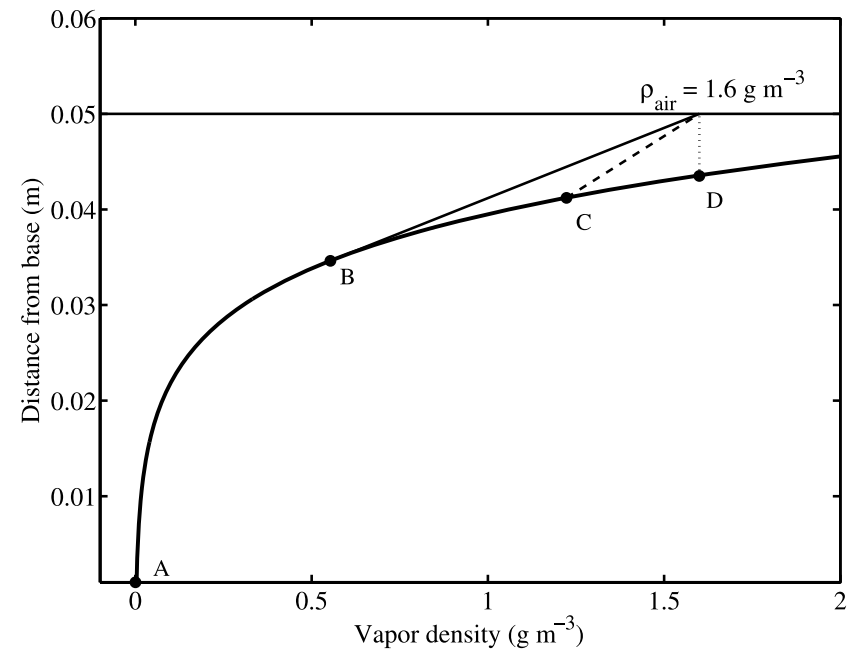

Figure 15. The steady state vapor density for a sample with a $13 \mathrm{~K} \mathrm{~cm}^{-1}$ temperature gradient. For levels where ice is present, the vapor density is set by the saturation vapor pressure of ice (thick curved line). For levels with no ice present, the gradient is linear (or approximately so) between the saturation line of the uppermost ice and the atmospheric vapor density, which is here set at $1.6 \mathrm{~g} \mathrm{~m}^{-3}$.

[1997] by the action of two parallel conductors where the contribution of ice in the intergrain space was proportional to the square root of the filling fraction, $\left(\sigma / \sigma_{0}\right)^{1 / 2}$ in our notation. This model exhibits a rapid increase in thermal conductivity for small filling fractions. The effect of the earliest deposited ice is significant with respect to its effect on the thermal properties of the regolith.

[81] As deposited ice increases the thermal conductivity of the regolith, heat conduction to depth becomes more efficient and the steep thermal gradients which can be developed in low conductivity, ice-free material are diminished. These lower thermal gradients result in shallower mean vapor density gradients which, as per equation (2), result in reduced ice deposition. In an environment such as Mars where the thermal gradient is not prescribed but instead can adjust to the changing thermal properties of the medium, the change in thermal conductivity acts in addition to ice content to reduce the ice accumulation rate. The effect of the thermal conductivity variation is strongest at small filling fractions where a minor change in ice content greatly affect the heat conduction in the bulk medium. On the other hand, the effect of ice content on deposition rate is greatest when filling fractions are larger.

\subsection{Ice Growth Physics and Phenomenology}

[82] In this experiment, the temperature gradient is static, while on a planetary surface, oscillating surface temperatures produce thermal waves which propagate into the subsurface. These oscillations are responsible, on diurnal and annual scales, for the pumping of $\mathrm{H}_{2} \mathrm{O}$ into and out of the subsurface. However, as shown by Schorghofer and Aharonson [2005], it is the mean annual vapor pressure which governs long-term ice evolution, the rapid oscillations of diurnal regolith breathing and adsorption being averaged out on this time scale. Thus, in the cartoon of Figure 15 and in the experimental setup, the time-averaged vapor density gradient is approximated by the vapor pressure curve resulting from a static linear temperature gradient. In such a time-averaged view, the density gradient between the ice table and the surface will appear linear since no net ice deposition occurs in this region.

[83] An initially dry soil subjected to a moist atmosphere will experience a rise in vapor density at all depths. If the vapor density increases to the saturation limit at some depth, ice will deposit; thereafter the vapor density remains fixed by the vapor pressure of the local ice. For depths where the vapor density is below the saturation limit, an unsaturated gradient will be established between the surface and the shallowest ice. This circumstance is shown schematically in Figure 15 as the line connecting the surface to point B.

[84] The ice present in the pore space beneath the ice table maintains saturation in the pore gas and higher maximum temperatures at shallow depths result in the down-directed time-averaged gradient. These temperature differences thus drive the diffusive movement of the water molecules. However, as the molecules move in response to this concentration gradient, they are still subject to collisions with the pore walls. Thus, changing ice content affects the flux of vapor even though the gradients and vapor densities are everywhere determined by the local temperatures.

[85] Equation (2) can be expanded as

$$
\frac{\partial \sigma}{\partial t}=\frac{\partial D}{\partial z} \frac{\partial \rho_{1}}{\partial z}+D \frac{\partial^{2} \rho_{1}}{\partial z^{2}} .
$$

Within the ice-free region, the time-averaged vapor density is linear, and so the second term of equation (18) is zero. The rate of net ice accumulation in this region is also zero, so the diffusion coefficient is also constant. Beneath the ice table, however, the vapor density gradient is nonlinear everywhere and, in a time-averaged view, always has the same sign, directing net vapor flux downward. Both terms of equation (18) contribute to the ice accumulation.

[86] The ice beneath the ice table accumulates, as observed in the experiments, in a continuous profile which implies a small value of $\partial D / \partial z$ within the ice-bearing region. The dominant term expressing the ice growth as a function of time is therefore the second term of equation (18), which indicates that the ice growth depends on the curvature of the vapor density profile (i.e., the divergence of the flux) and the local value of the diffusion coefficient.

[87] In Figure 15, the gradients in vapor density are equal across the interface at the depth indicated by point B (curves in the ice-bearing and ice-free regions are tangent). At early times, little ice will have deposited and the diffusion coefficients in the ice-free and ice-bearing regions will also be equal. Hence, the fluxes across the ice table will be the same.

[88] If modification of $D$ by ice deposition did not occur, the balance in fluxes between the ice-free and ice-bearing regions would persist and the ice table would not move from the depth indicated by point B. But ice deposition reduces the diffusion coefficient at the ice table (and depths below), thus reducing the flux into the ice-bearing soil. The gradient in the ice-free region is not directly affected by the diffusion process and so continues to supply water molecules to the ice table. The imbalance of fluxes results in an 
increase in the local vapor density above the ice table, eventually reaching saturation and depositing ice. The ice table therefore moves toward its equilibrium depth (such an intermediate step is indicated by point $\mathrm{C}$ in Figure 15).

[89] The time-averaged vapor density gradient in this shallower ice is higher than the gradients beneath, again because of the higher maximum temperatures. But the mean gradient through the ice-free region is smaller for the shallower, warmer ice. The flux to the ice table is thus reduced as it migrates to shallower depths. The high gradients in vapor density drive the ice to accumulate more quickly at shallower depths, but this accumulation is limited by the flux of available water. The density gradient between the atmosphere and the ice table becomes progressively smaller and smaller, resulting in an asymptotic approach to the equilibrium depth.

[90] At equilibrium, indicated in Figure 15 by point $\mathrm{D}$, inward flux must be zero. This can obtain if either the gradient between the surface and the ice table is zero, or the diffusion coefficient at the ice table is zero. However, the time-averaged vapor density gradient in the ice-bearing region continues to drive water vapor down-gradient to deeper depths. Water vapor surrounded above and below by ice experiences only the local vapor gradient due to temperature; it is independent of the atmospheric density. Since ice beneath the ice table is continuously redistributing down gradient, there will remain a positive net flux into the subsurface if the equilibrium depth is above the ice table. High ice contents permit only small fluxes within the icerich region, allowing the ice table to be close to the equilibrium depth when filling fractions are high. If the climate on Mars has been stable enough to permit substantial filling, differences between equilibrium predictions of ice table depth [e.g., Mellon et al., 2004; Schorghofer and Aharonson, 2005] and observed depths (within the accuracy achievable by spacecraft such as Mars Odyssey and the Phoenix Lander) are unlikely to be large.

[91] This difference between the equilibrium depth and the ice table would persist so long as some accessible volume of the porous medium is unsaturated. Oscillating temperatures and atmospheric humidities unresolved in this annually averaged view permits excess ice to deposit in favorable seasons. Were transient ice able to completely saturate the pore spaces at the ice table, the resulting choked layer would be short lived. The ice would be removed from two directions: to the atmosphere as the overlying transient ice disappeared, and into the deeper, unsaturated regolith along the down-directed vapor density gradient.

[92] If the atmosphere were to become drier and the equilibrium depth receded beneath the ice table, the cryosphere would exhibit net loss of ice from the top of the ice table. At the same time, however, there would be a continued redistribution of deeper ice within the regolith. Ice beneath the ice table would migrate down the timeaveraged vapor density gradient until all ice above it was removed and it was able to feel the effect of the vapor density gradient (now reversed in sign) in the ice-free region above.

\subsection{High-Volume Ice on Mars}

[93] Two mechanisms by which significant volumes of ice can be emplaced in the shallow subsurface of Mars are
(1) precipitation and subsequent burial and (2) inward diffusion of atmospherically derived vapor. The former process requires climate conditions significantly different from those currently observed, while the latter may have operated frequently in the past in addition to being active today.

[94] Most unconsolidated sandy terrestrial soils have porosities between 30-50\% [Taylor, 1977], though finer particulates can give rise to porosities up to $60 \%$ [Baver, 1940]. The Gamma Ray Spectrometer Suite on board Mars Odyssey measured middle- to high-latitude hydrogen abundances equivalent to up to $70 \%$ ice by volume [Prettyman et al., 2004]. Ice contents of this magnitude would require precipitation and burial, the subsurface growth of pure ice lenses, or unusually high initial porosities which may be filled by diffusion.

[95] The presence of polygonal terrain on Mars shows that a mechanism capable of moving soil grains has been active in these regions. Liquid water films, an important component of ice wedge formation on Earth [Konrad and Morgenstern, 1980; Konrad and Duquennoi, 1993; Dash et al., 1995; Wettlaufer, 1999], are not favored on Mars at present. Thermal contraction cracking [Mellon et al., 1997] may play a dominant role in the dynamics of presently forming polygons [Mellon et al., 1997]. We do not observe evidence of heaving, lensing, or grain segregation in our static environment experimental setup, but this is not surprising given the experimental length scales and absence of a thermal oscillation. It should also be noted that microscopic effects of grain movement accumulate over long times, and cracking due to the stress of thermal contraction require distances on the order of a polygon diameter (tens to hundreds of meters) [Mangold, 2005], much greater than the times and length scales characteristic of these experiments.

[96] Fisher [2005] suggests that thermal cracking of solid (perhaps interstitial) ice due to seasonal temperature variations may serve to keep deep regolith more open to vapor transport despite constrictive flux reduction in shallow layers. Cracks could penetrate into an otherwise ice-saturated soil, opening up further volume for subsurface deposition. Ice wedges on Earth form through the trapping of seasonal meltwater in the thermal cracks. The absence of a liquid phase on present-day Mars would tend to favor sand wedge formation instead. But vapor propagation through the space opened by the crack would be enhanced over porous medium diffusion, and material at the base of the crack is shielded from direct insolation and will therefore remain cold relative to the surface. Ice wedges on Mars may therefore arise from vapor diffusion processes. If the combined volume of the vertically penetrating ice veins arising from such cracks is large, they could contribute to high observed ice contents.

\subsection{Phoenix Mars lander}

[97] The Phoenix Mars Lander has landed in the north polar region of Mars where equilibrium models and neutron spectrometer data from the Mars Odyssey spacecraft predict the presence of subsurface ice within centimeters of the surface [Boynton et al., 2006; Arvidson et al., 2008; Mellon et al., 2008]. The lander is equipped with a robotic arm which has trenched into relatively unconsolidated surface 
regolith; excavation down to the ice table, one of the primary mission goals, has been achieved [Arvidson et al., 2008]. In so doing, Phoenix data will be able to provide a number of ground truths regarding the behavior of subsurface ice on Mars.

[98] The robotic arm has limited mechanical strength and leverage and may be unable to penetrate deeply through soil with a high ice content, or through pure ice if it is not highly porous. Despite this limitation, the indications are that an abrupt change in mechanical strength of the regolith demonstrates the existence of a sharp ice table as predicted theoretically and as has been seen in our experiments. Using the rasping tool at the scoop, Phoenix will attempt to remove some surface layers of the shallow ice table. Photographic analysis of these raspings and the divots made in the ice table, as well as planned delivery of icy regolith to various on-deck instruments, may constrain the soil-to-ice mixing ratio. This could illuminate whether this ice was diffusively derived, as would be suggested by a low to moderate ice-to-soil ratio, or was emplaced by precipitation and subsequent burial, consistent with a high ratio.

[99] The morphology of the ice table could be correlated with the original surface albedo and rock distribution. Though it is not equipped with a brush to remove unconsolidated debris, a number of subsurface profiles acquired during trenching activities may allow a coarse three-dimensional map of the ice table to be developed.

[100] Low mechanical strength of the ice-bearing soil layers would be indicative of filling fractions less than $100 \%$. Though not equipped with means of directly determining water content, data from Phoenix instruments such as the Thermal and Evolved Gas Analyzer (TEGA) and the Thermal and Electrical Conductivity Probe (TECP) may be able to constrain this quantity. Incomplete filling would be indicative of a nonequilibrium state which is continuing to evolve over time, constraining the history of the temperature and atmospheric water content at the landing site. Combined data sets from measurements of soil thermal conductivity and optical microscope images of the regolith particles will help constrain other soil properties, such as diffusivity, which are necessary inputs to understanding the history of subsurface ice.

\section{Summary}

[101] We have used a Mars environment chamber to demonstrate the diffusive filling of regolith pore spaces from atmospherically derived water vapor in the absence of a bulk liquid phase. This allows the first examination of bulk ice deposition via vapor diffusion at low pressures in the laboratory. We study this process at three scales.

[102] At the scale of individual soil grains, optical micrographs reveal preferential deposition at grain contact points, forming ice necks in close association with regolith particles which should significantly affect the thermal conductivity. Much of the deposited ice is turbid, suggesting the presence of grain boundaries, vacancies, or trapped gases within the ice which would reduce its bulk density. Bubbles on the order of 10-20 $\mu \mathrm{m}$ across have been observed trapped in the ice, suggesting that ice on Mars deposited through vapor diffusion may be a candidate for the extraction of trapped atmospheric gases.
[103] At the scale of the ice table, measurements of the transition depth have revealed the sharpness and shape of the contact. The transition between well-cemented and completely unconsolidated grains is on the order of a single grain diameter $(\sim 500 \mu \mathrm{m})$. The morphology of the interface is sensitive to the local temperature field and is affected by surface irregularities such as heat pipes (e.g., a warm probe head or a rock) and albedo variations (e.g., coated disks or dust patches), and the magnitude of these perturbations decays with depth. The ice table follows temperature isotherms, which parallel the surface unless perturbations arising from, for example, albedo differences, deflect the temperature field.

[104] Finally, at the deeper scale of the "cryosphere," i.e., the ice beneath the interface, the quantity and distribution of ice as a function of depth and time has been revealed through gravimetric water content analysis. A series of experiments and numerical models of differing atmospheric vapor densities illustrate the sensitivity of ice table growth rate to the humidity of the atmosphere, and the insensitivity of ice at depths beneath the ice table to this same quantity. The evolution of the cryospheric ice beneath the ice table depends only on the local temperature gradient.

[105] The deposition of ice significantly reduces subsequent deposition rates via a process of pore space constriction. Numerical models of the experimental conditions which incorporate constriction capture the major effects of that process with a parameterized modification of the diffusion coefficient proportional to filling fraction. Although some observed differences between experiments and models may involve a lower density of deposited ice, our observations of $90 \%$ filling after $530 \mathrm{~h}$ show that the maximum density is at least this much. At intermediate filling, we find a stronger obstruction than linear in filling fraction. The simultaneous reduction of available porosity and of molecular hop length (i.e., the increasing frequency of collisions with pore walls) as ice is deposited results in an expected dependence of $D$ proportional to the square of the filling fraction, consistent with measurements when the filling fraction is high. The tortuosity may also change at moderate to high filling fractions, further increasing the sensitivity of $D$ to $\sigma$. Further investigation is needed to describe the behavior of the system at high filling fractions.

[106] The numerical model is used to examine long-term growth effects under simulated temperature and humidity conditions. The effect of increased ice content asymptotically diminishes the vapor flux beneath the ice table and similarly retards the ice deposition rate.

[107] The complete filling of the regolith with atmospheric vapor on obliquity-scale times will be inhibited by reduced rates of ice deposition due to the phenomena observed here, but complete choking will not occur unless the pore volume is completely saturated with ice. Lack of complete filling in a regolith column implies a nonequilibrium condition such that the quantity and distribution of subsurface ice is continually evolving. These studies will inform observations of subsurface ice by Phoenix and subsequent spacecraft addressing similar regions.

[108] Acknowledgments. We gratefully acknowledge Kenny Oslund and Matt Siegler for their assistance in developing and testing the 
experimental apparatus used here. This work was funded in part by NASA's Mars Fundamental Research Program.

\section{References}

Arvidson, R., et al. (2008), Mars Exploration Program 2007 Phoenix landing site selection and characteristics, J. Geophys. Res., 113, E00A03, doi:10.1029/2007JE003021.

Baver, L. D. (1940), Soil structure, in Soil Physics, chap V, pp. 160-167, John Wiley, New York.

Bockheim, J. G. (2002), Landform and soil development in the McMurdo Dry Valleys, Antarctica: A regional synthesis, Arct. Antarct. Alp. Res., 34, 308-317, doi: $10.2307 / 1552489$.

Boynton, W., et al. (2002), Distribution of hydrogen in the near-surface of Mars: Evidence for subsurface ice deposits, Science, 297(5578), $81-85$.

Boynton, W. V., D. M. Janes, M. J. Finch, and R. M. S. Wiliams (2006), Simultaneous determination of dry-layer thickness and sub-surface ice content in the polar regions of Mars: Implications for the Phoenix landing site selection, Lunar Planet. Sci., XXXVII, abstract 2736.

Carr, M. (1996), Water on Mars, 229 pp., Oxford Univ. Press, New York. Chapman, S., and T. Cowling (1970), The Mathematical Theory of NonUniform Gases, 3rd ed., Cambridge Univ. Press, Cambridge, U. K.

Clifford, S., and D. Hillel (1983), The stability of ground ice in the equatorial region of Mars, J. Geophys. Res., 88(B3), 2456-2474.

Clifford, S., and D. Hillel (1986), Knudsen diffusion: The effect of small pore size and low gas pressure on gaseous transport in soil, Soil Sci. 141(4), 289-297.

Dash, J., H. Fu, and J. Wettlaufer (1995), The premelting of ice and its environmental consequences, Rep. Prog. Phys., 58, 115-167.

Doran, P. T., C. P. McKay, G. D. Clow, G. L. Dana, A. G. Fountain, T. Nylen, and W. B. Lyons (2002), Valley floor climate observations from the McMurdo dry valleys, Antarctica, 1986-2000, J. Geophys. Res., 107(D24), 4772, doi:10.1029/2001JD002045.

Elbaum, M., S. G. Lipson, and J. G. Dash (1993), Optical study of surface melting on ice, J. Cryst. Growth, 129, 491-505, doi:10.1016/00220248(93)90483-D.

Evans, R., G. Watson, and E. A. Mason (1961), Gaseous diffusion in porous media at uniform pressure, J. Chem. Phys., 35(6), 2076-2083.

Feldman, W. C., M. T. Mellon, O. Gasnault, B. Diez, R. C. Elphic, J. J. Hagerty, D. J. Lawrence, S. Maurice, and T. H. Prettyman (2007), Vertical distribution of hydrogen at high northern latitudes on Mars: The Mars Odyssey Neutron Spectrometer, Geophys. Res. Lett., 34, L05201, doi:10.1029/2006GL028936.

Fisher, D. A. (2005), A process to make massive ice in the martian regolith using long-term diffusion and thermal cracking, Icarus, 179, 387-397, doi:10.1016/j.icarus.2005.07.024.

Ham, J. M., and E. J. Benson (2004), On the construction and calibration of dual-probe heat capacity sensors, Soil Sci. Soc. Am. J., 68, 1185-1190.

Hardy, B. (1998), Its-90 formulations for vapor pressure, frostpoint, temperature dewpoint temperature, and enhancement factors in the range -100 to $+100 \mathrm{c}$, in Proceedings of the Third International Symposium on Humidity and Moisture, edited by B. Hardy, pp. 1-15, Natl. Phys. Lab., London.

Head, J. W., J. F. Mustard, M. A. Kreslavsky, R. E. Milliken, and D. R. Marchant (2003), Recent ice ages on Mars, Nature, 426, 797-802.

Hindmarsh, R., F. Van der Wateren, and A. Verbers (1998), Sublimation of ice through sediment in Beacon Valley, Antarctica, Geogr. Ann., Ser. A, Phys. Geogr, 80(3-4), 209-219.

Hobbs, P. V., and B. J. Mason (1964), The sintering and adhesion of ice, Philos. Mag., 9(98), 181-197, doi:10.1080/14786436408229184.

Hudson, T. L., and O. Aharonson (2008), Diffusion barriers at Mars surface conditions: Salt crusts, particle size mixtures, and dust, J. Geophys. Res., 113, E09008, doi:10.1029/2007JE003026.

Hudson, T., O. Aharonson, N. Schorghofer, C. Farmer, M. Hecht, and N. Bridges (2007), Water vapor diffusion in Mars subsurface environments, J. Geophys. Res., 112, E05016, doi:10.1029/2006JE002815.
Konrad, J.-M., and C. Duquennoi (1993), A model of water transport and ice lensing in freezing soils, Water Resour. Res., 29(9), 3109-3124.

Konrad, J.-M., and N. R. Morgenstern (1980), The segregation potential of a freezing soil, Can. Geotech. J., 18, 482-491.

Landau, L., and E. Lifshitz (1987), Fluid Mechanics, Pergamon, Oxford, U. K.

Leighton, R., and B. Murray (1966), Behavior of carbon dioxide and other volatiles on Mars, Science, 153, 136-144.

Litvak, M. L., et al. (2006), Comparison between polar regions of Mars from HEND/Odyssey data, Icarus, 180, 23-37.

Mangold, N. (2005), High latitude patterned grounds on Mars: Classification, distribution, and climatic control, Icarus, 174, 336-359, doi:10.1016/j.icarus.2004.07.030.

McKay, C. P., M. T. Mellon, and E. I. Friedmann (1998), Soil temperatures and stability of ice-cemented ground in the McMurdo Dry Valleys, Antarctica, Antarct. Sci., 10(1), 31-38.

Mellon, M., and B. Jakosky (1993), Geographic variations in the thermal and diffusive stability of ground ice on Mars, J. Geophys. Res., 98(E2), $3345-3364$

Mellon, M., and B. Jakosky (1995), The distribution and behavior of Martian ground ice during past and present epochs, J. Geophys. Res., 100(E6), 11,781-11,799.

Mellon, M., B. Jakosky, and S. Postawko (1997), The persistence of equatorial ground ice on Mars, J. Geophys. Res., 102(E8), 19,357-19,369.

Mellon, M., W. Feldman, and T. Prettyman (2004), The presence and stability of ground ice in the southern hemisphere of Mars, Icarus, 169(2), 324-340, doi:10.1016/j.icarus.2003.10.022.

Mellon, M. T., W. V. Boynton, W. C. Feldman, R. E. Arvidson, T. N. Titus, J. L. Bandfield, N. E. Putzig, and H. G. Sizemore (2008), A prelanding assessment of the ice table depth and ground ice characteristics in Martian permafrost at the Phoenix landing site, J. Geophys. Res., 113, E00A25, doi:10.1029/2007JE003067.

Paige, D. (1992), The thermal stability of near-surface ground ice on Mars, Nature, 356(6364), 43-45.

Pollard, W., and R. Present (1948), On gaseous self-diffuson in long capillary tubes, Phys. Rev., 73, 762-774.

Prettyman, T. H., et al. (2004), Composition and structure of the Martian surface at high southern latitudes from neutron spectroscopy, J. Geophys. Res., 109, E05001, doi:10.1029/2003JE002139.

Schorghofer, N. (2005), A physical mechanism for long-term survival of ground ice in Beacon Valley, Antarctica, Geophys. Res. Lett., 32, L19503, doi:10.1029/2005GL023881.

Schorghofer, N. (2007), Dynamics of ice ages on Mars, Nature, 449(7159), 192-194, doi:10.1038/nature06082.

Schorghofer, N., and O. Aharonson (2005), Stability and exchange of subsurface ice on Mars, J. Geophys. Res., 110, E05003, doi:10.1029/ 2004JE002350.

Sizemore, H. G., and M. T. Mellon (2006), Effects of soil heterogeneity on Martian ground-ice stability and orbital estimates of ice table depth, Icarus, 185, 269-358.

Sugden, D., D. Marchant, N. Potter, R. Souchez, G. Denton, C. Swisher, and J. Tison (1995), Preservation of Miocene glacier ice in East Antarctica, Nature, 376(6539), 412-414

Taylor, J. C. M. (1977), Sandstones as reservoir rocks, Developments in Petroleum Geology, vol. 1, edited by G. D. Hobson, chap. 5, pp. 147196, Appl. Sci., London.

Wettlaufer, J. S. (1999), Ice surfaces: Macroscopic effects of microscopic structure, Philos. Trans. R. Soc. London, Ser. A, 357, 3403-3425.

O. Aharonson and T. Hudson, Division of Geological and Planetary Sciences, California Institute of Technology, Pasadena, CA 91125, USA. (thudson@gps.caltech.edu)

N. Schorghofer, Institute for Astronomy, University of Hawai' $i$ at Mānoa, Honolulu, HI 96822, USA. 